\title{
Optimal Dividend and Capital Injection Strategies in the Cramér-Lundberg Risk Model
}

\author{
Yan $\mathrm{Li}^{1}$ and Guoxin $\mathrm{Liu}^{2}$ \\ ${ }^{1}$ School of Insurance and Economics, University of International Business and Economics, Beijing 100029, China \\ ${ }^{2}$ Shijiazhuang Tiedao University, Shijiazhuang 050043, China
}

Correspondence should be addressed to Yan Li; email.liyan@163.com

Received 12 March 2014; Revised 25 August 2014; Accepted 15 September 2014

Academic Editor: Guangchen Wang

Copyright (C) 2015 Y. Li and G. Liu. This is an open access article distributed under the Creative Commons Attribution License, which permits unrestricted use, distribution, and reproduction in any medium, provided the original work is properly cited.

\begin{abstract}
We discuss the optimal dividend and capital injection strategies in the Cramér-Lundberg risk model. The value function $V(x)$ is defined by maximizing the discounted value of the dividend payment minus the penalized discounted capital injection until the time of ruin. It is shown that $V(x)$ can be characterized by the Hamilton-Jacobi-Bellman equation. We find the optimal dividend barrier $b$, the optimal upper capital injection barrier 0 , and the optimal lower capital injection barrier $-z^{*}$. In the case of exponential claim size especially, we give an explicit procedure to obtain $b,-z^{*}$, and the value function $V(x)$.
\end{abstract}

\section{Introduction}

In the modern theory of risk, people tend to study the cost of postponing or avoiding outright ruin; that is, ruin does not mean the end of the game but only the necessity of raising additional money. So the risk process can continue if there is a suitable injection of surplus.

Borch [1] pointed out that it was a good investment to rescue an insolvent insurance company, provided that its deficit was not too large. He studied this problem for a random walk model and suggested that the company should be rescued only if the deficit was smaller than the expected profits from the rescue operation.

For a diffusion model, Sethi and Taksar [2] considered the problem of finding an optimal financing mix of retained earnings and external equity for maximizing the value of a corporation. They showed that the optimal policy can be characterized in terms of two threshold parameters. Løkka and Zervos [3] studied the same problem with possibility of bankruptcy in a model of Brownian motion with drift. Depending on the relationships between the coefficients, the optimal strategy requires the consideration of two auxiliary suboptimal models. For more references in diffusion model see He and Liang $[4,5]$, and so forth.
As pointed out by Bäuerle [6], the classical approach is to model the liquid assets or risk reserve process of the insurance company as a piecewise deterministic Markov process (PDMP). However, within this setting the control problem is very hard and many characteristics of the risk process can not be calculated in closed form.

For the Cramér-Lundberg risk model without bankruptcy (i.e., the shareholders will inject capital to cover the deficit whatever serious it is) the optimal dividend problem was studied. See, for example, Dickson and Waters [7], Gerber et al. [8], Kulenko and Schmidli [9], and so forth. This capital injection strategy makes sense for itself; at the same time we notice that the injected capital can be viewed as an investment. Therefore the shareholders should consider the return of it. If the injected amount is small enough to the shareholders to earn positive net profit, they accept to do so and survive the company. Otherwise, they will refuse to inject capital anymore and ruin occurs. So what is the optimal capital injection strategy is worth to be discussed.

In this paper, we will discuss the optimal dividend payment and capital injection strategies in the Cramér-Lundberg risk model. The objective is to maximize the discounted dividends payments minus the penalized discounted capital injections. Through the discussion of the optimal capital 
injection strategy, we find the maximal deficit which the shareholders can bear. Moreover, from the mathematical point of view we give a rigorous proof that it is optimal to inject capital once the reserves are below 0 , that is, the moment ruin occurs (in the previous literature about capital injection strategy, considering discounting, it could not be optimal to inject capital before it is really necessary. Therefore, the shareholders postpone the injection as long as possible and just conjecture that it is optimal to do so when the reserves become 0 ).

Suppose the reserve process of an insurance company at time $t$ is

$$
X_{t}=x+c t-\sum_{i=1}^{N_{t}} Y_{i}
$$

where $x \in R$ is the initial capital, $c>0$ is the premium rate, $\left\{N_{t}, t \geq 0\right\}$ is a Poisson process with intensity $\lambda>$ 0 , and $\left\{Y_{i}, i \geq 1\right\}$ is a sequence of strictly positive i.i.d. random variables with the distribution function $F(x)$. In addition, $\left\{Y_{i}, i \geq 1\right\}$ and $\left\{N_{t}, t \geq 0\right\}$ are independent. We assume that $E Y_{i}=\mu<\infty$ and $F(x)$ is continuous. $\left\{X_{t}\right\}$ is on a filtrated probability space $\left(\Omega, \mathscr{F},\left\{\mathscr{F}_{t}\right\}_{t \geq 0}, P\right)$, where $\left\{\mathscr{F}_{t}\right\}_{t \geq 0}$ is the smallest right-continuous filtration such that $\left\{X_{t}\right\}$ is adapted. Let $P_{x}$ and $E_{x}$ denote the probability and the expectation with initial capital $x$, respectively.

Now we enrich the model with a strategy $\pi=\left\{\left(D_{t}, Z_{t}\right)\right\}$. $\left\{D_{t}\right\}$ and $\left\{Z_{t}\right\}$ denote the aggregate dividends and capital injections paid up to time $t$, respectively. The strategy $\pi$ is admissible if

(1) $\left\{D_{t}\right\}$ is càdlàg, increasing and adapted processes with $D_{0-}=0$;

(2) $\left\{Z_{t}\right\}$ is càglàd, increasing and adapted processes with $Z_{0}=0$.

The reserve turns to

$$
X_{t}^{\pi}=X_{t}-D_{t}+Z_{t}
$$

Since the strategy $\pi$ will not assure that the process $\left\{X_{t}^{\pi}\right\}$ is always larger than 0 , ruin is possible. The ruin time is defined by

$$
T^{\pi}=\inf \left\{t \geq 0, X_{t+}^{\pi}<0\right\}
$$

The value of a strategy $\pi$ is

$$
V^{\pi}(x)=E_{x}\left[\int_{0-}^{T^{\pi}-} e^{-\delta t} \mathrm{~d} D_{t}-\phi \int_{0}^{T^{\pi}} e^{-\delta t} \mathrm{~d} Z_{t}\right],
$$

where $\delta>0$ is a discounted factor and $\phi>1$ is a penalizing factor. The point 0 being included in the integration area is for the reason of taking an immediate dividend $D_{0}>0$ into the value. Our purpose is to maximize $V^{\pi}(x)$. The value function is defined by

$$
V(x)=\sup _{\pi \in \Pi} V^{\pi}(x)
$$

where $\Pi$ denotes the set of all admissible strategies.
The paper is organized as follows. In Section 2, the dividend strategy is constrained by a restricted density. Some properties of the value function $V(x)$ are proved. We show that $V(x)$ can be characterized by the Hamilton-JacobiBellman equation. Moreover, if $V(x)$ is concave, the optimal dividend and capital injection strategies are both barrier strategies. If we remove the constraint on the dividend strategy, the results on $V(x)$ and optimal strategies are extended in Section 3. In the last section, we give an explicit procedure to obtain the optimal dividend barrier $b$, the optimal lower capital injection barrier $-z^{*}$, and the value function $V(x)$ when the claim size is exponentially distributed.

\section{Dividends with Restricted Densities}

In this section, we study this optimization problem under the constraint that the dividends are paid at a dividend rate, which is bounded by a positive constant $u_{0}$; that is, $0 \leq U_{t} \leq$ $u_{0}<\infty$. Then $D_{t}=\int_{0}^{t} U_{s} \mathrm{~d} s$ and

$$
V^{\pi}(x)=E_{x}\left[\int_{0}^{T^{\pi}} e^{-\delta t} U_{t} \mathrm{~d} t-\phi \int_{0}^{T^{\pi}} e^{-\delta t} \mathrm{~d} Z_{t}\right] .
$$

In this section, $\Pi^{r}$ denotes the set of all admissible restricted strategies and $\pi=\left(U_{t}, Z_{t}\right)$. So the value function

$$
V(x)=\sup _{\pi \in \Pi^{r}} V^{\pi}(x) .
$$

2.1. The Value Function $V(x) . V(x)$ has the following properties.

Lemma 1. If the capital injection strategy is defined by

$$
Z_{t}=\max \left\{-\inf _{0 \leq s<t}\left(X_{s}-D_{s}\right), 0\right\},
$$

Then, for $x \in R_{+}$, the value under any dividend strategy $\left\{D_{t}\right\}$ is bounded from below by $-\phi \lambda \mu / \delta$.

Proof. Under this assumption, ruin time is $\infty$. The maximal amount of capital injection may be that the shareholders cover all the claims. If we are not considering the dividends, value under such a strategy is the worst one. Using the time of the $k$ th claim $T_{k}$ is Gamma $\Gamma(\lambda, k)$, so

$$
E\left[\sum_{k=1}^{\infty} Y_{k} e^{-\delta T_{k}}\right]=\mu \sum_{k=1}^{\infty}\left(\frac{\lambda}{\lambda+\delta}\right)^{k}=\frac{\lambda \mu}{\delta} .
$$

The value is bounded from below by $-\phi \lambda \mu / \delta$.

Lemma 2. $V(x)$ is increasing and Lipschitz continuous on $(-\infty, \infty)$. Moreover, $0 \leq V(x) \leq u_{0} / \delta$ and $\lim _{x \rightarrow \infty} V(x)=$ $u_{0} / \delta$.

Proof. Obviously, $V(x)$ is increasing. For $x<0$, if define the strategy $\pi$ as $Z_{t}=U_{t}=0$, then $V(x) \geq V^{\pi}(x)=0$. Because $V(x)$ is increasing, $V(x) \geq 0$ for $x \in R$. If $U_{t}=u_{0}, Z_{t}=0$, then

$$
V(x) \leq \int_{0}^{\infty} u_{0} e^{-\delta t} \mathrm{~d} t=\frac{u_{0}}{\delta}
$$


Consider a strategy $\pi=\left(U_{t}, Z_{t}\right)$, where $U_{t}=u_{0}$ and $Z_{t}=$ $\max \left\{-\inf _{0 \leq s<t}\left(X_{s}-D_{s}\right), 0\right\}$. Then $T^{\pi}=\infty$. Define $\tau_{x}^{\pi}=\inf \{t$ : $\left.x+\left(c-u_{0}\right) t-\sum_{i=1}^{N_{t}} Y_{i}<0\right\}$. Using Lemma 1,

$$
\begin{aligned}
\int_{0}^{T^{\pi}} e^{-\delta t} \mathrm{~d} Z_{t} & =\int_{0}^{\infty} e^{-\delta t} \mathrm{~d} Z_{t}=\int_{\tau_{x}^{\pi}}^{\infty} e^{-\delta t} \mathrm{~d} Z_{t} \\
& =e^{-\delta \tau_{x}^{\pi}} \int_{0}^{\infty} e^{-\delta t} \mathrm{~d} Z_{t+\tau_{x}^{\pi}} \leq e^{-\delta \tau_{x}^{\pi}} \phi \frac{\lambda \mu}{\delta} .
\end{aligned}
$$

When $x \rightarrow \infty$, then $\tau_{x}^{\pi} \rightarrow \infty$ and $P_{x}\left(\int_{0}^{\infty} e^{-\delta t} \mathrm{~d} Z_{t}>\varepsilon\right) \rightarrow$ 0 . So we have

$$
\begin{aligned}
V(x) & \geq V^{\pi}(x) \\
& \geq E\left[\int_{0}^{\tau_{x}^{\pi}} u_{0} e^{-\delta t} \mathrm{~d} t-\phi \int_{0}^{\infty} e^{-\delta t} \mathrm{~d} Z_{t}\right] \rightarrow \frac{u_{0}}{\delta} .
\end{aligned}
$$

Combining with (10), we have $\lim _{x \rightarrow \infty} V(x)=u_{0} / \delta$.

For $x \geq 0$, let $h>0$ be small. Define

$$
\begin{aligned}
& U_{t}= \begin{cases}0, & \text { if } 0 \leq t<h \wedge T_{1}, \\
\widetilde{U}_{t-h}, & \text { if } t \wedge T_{1} \geq h, \\
0, & \text { if } T_{1}<h,\end{cases} \\
& Z_{t}= \begin{cases}0, & \text { if } 0 \leq t<h \wedge T_{1}, \\
\widetilde{Z}_{t-h}, & \text { if } t \wedge T_{1} \geq h, \\
0, & \text { if } T_{1}<h,\end{cases}
\end{aligned}
$$

where $\widetilde{\pi}=(\widetilde{U}, \widetilde{Z}) \in \Pi^{r}$ is for the initial capital $x+c h$. While $P\left(T_{1} \geq h\right)=e^{-\lambda h}$, then

$$
\begin{aligned}
V(x) \geq & V^{\pi}(x) \\
= & E\left[E\left[\int_{0}^{T^{\pi}} e^{-\delta t} U_{t} \mathrm{~d} t-\phi \int_{0}^{T^{\pi}} e^{-\delta t} \mathrm{~d} Z_{t} \mid T_{1}\right]\right] \\
= & P\left(T_{1} \geq h\right) \\
& \times E\left[\int_{h}^{T^{\pi}} e^{-\delta t} U_{t} \mathrm{~d} t-\phi \int_{h}^{T^{\pi}} e^{-\delta t} \mathrm{~d} Z_{t} \mid T_{1} \geq h\right] \\
= & e^{-\lambda h} E\left[E\left[\int_{h}^{T^{\pi}} e^{-\delta t} U_{t} \mathrm{~d} t-\phi \int_{h}^{T^{\pi}} e^{-\delta t} \mathrm{~d} Z_{t} \mid \mathscr{F}_{h}\right]\right] \\
= & e^{-\lambda h} E\left[e ^ { - \delta h } E \left[\int_{0}^{T^{\pi}-h} e^{-\delta t} U_{t+h} \mathrm{~d} t\right.\right. \\
= & \left.e^{-(\lambda+\delta) h} E\left[\int_{0}^{T^{\pi}-h} e_{0}^{T^{\pi}-h t} e^{-\delta t} \mathrm{~d} Z_{t+h} \mid \mathscr{F}_{h}\right]\right] \\
= & e^{-(\lambda+\delta) h} V^{\tilde{\pi}}(x+c h)
\end{aligned}
$$

and so

$$
V(x) \geq \sup _{\tilde{\pi} \in \Pi^{r}} e^{-(\lambda+\delta) h} V^{\tilde{\pi}}(x+c h)=e^{-(\lambda+\delta) h} V(x+c h) .
$$

From the bounded property of $V(x)$, we have

$$
\begin{aligned}
0 & \leq V(x+c h)-V(x) \leq V(x+c h)\left(1-e^{-(\lambda+\delta) h}\right) \\
& \leq V(x+c h)(\lambda+\delta) h \leq \frac{u_{0}}{\delta}(\lambda+\delta) h .
\end{aligned}
$$

Let the shareholder inject $h$ and follow the optimal strategy afterwards when $x<0$. So $V(x) \geq V(x+h)-\phi h$; that is,

$$
V(x+h)-V(x) \leq \phi h .
$$

Thus $V(x)$ is Lipschitz continuous on $(-\infty, \infty)$.

2.2. HJB Equation and the Optimal Strategy. In this section, we will derive the HJB equation satisfied by the value function $V(x)$ and discuss the optimal strategy $\pi^{*}$.

Similar to the discussion in Azcue and Muler [10], the following dynamic programming principle holds:

$$
\begin{aligned}
V(x)=\sup _{\pi} E_{x} & {\left[\int_{0}^{\tau \wedge T^{\pi}} e^{-\delta t} U_{t} \mathrm{~d} t\right.} \\
& \left.-\phi \int_{0}^{\tau \wedge T^{\pi}} e^{-\delta t} \mathrm{~d} Z_{t}+e^{-\delta\left(\tau \wedge T^{\pi}\right)} V\left(X_{\tau \wedge T^{\pi}}^{\pi}\right)\right]
\end{aligned}
$$

for $x \in R_{+}$and any $\left\{\mathscr{F}_{t}\right\}$-stopping time $\tau$. This principle may serve us to derive the HJB equation.

For $x \geq 0, \varepsilon>0$, and any admissible strategy $\pi$, define $\sigma^{\pi}=\inf \left\{t \geq 0, X_{t}^{\pi} \notin(x-\varepsilon, x+\varepsilon)\right\}$. Choose $\varepsilon$ small enough; then $\sigma^{\pi}<T^{\pi}$. Let $\tau^{\pi}=\sigma^{\pi} \wedge h, h>0$. So $\tau^{\pi} \rightarrow 0$ a.s. $h \rightarrow 0$. Applying Itô formula into $e^{-\delta \tau^{\pi}} V\left(X_{\tau^{\pi}}^{\pi}\right)$, we have

$$
\begin{aligned}
e^{-\delta \tau^{\pi}} & V\left(X_{\tau^{\pi}}^{\pi}\right) \\
= & V\left(X_{0}^{\pi}\right) \\
& +\int_{0}^{\tau^{\pi}} e^{-\delta s}\left(c-U_{s}\right) V^{\prime}\left(X_{s^{-}}^{\pi}\right)-\delta e^{-\delta s} V\left(X_{s-}^{\pi}\right) \mathrm{d} s \\
& +\sum_{\substack{0 \leq s \leq \tau^{\pi} \\
X_{s-}^{\pi} \neq X_{s}^{\pi}}} e^{-\delta s}\left[V\left(X_{s}^{\pi}\right)-V\left(X_{s^{-}}^{\pi}\right)\right] \\
& +\sum_{\substack{0 \leq s<\tau^{\pi} \\
X_{s}^{\pi} \neq X_{s+}^{\pi}}} e^{-\delta s}\left[V\left(X_{s+}^{\pi}\right)-V\left(X_{s}^{\pi}\right)\right] .
\end{aligned}
$$

If $U_{t}>c,\left\{X_{t}^{\pi}\right\}$ could become negative before the first claim and so dividends lead to ruin. Considering the early penalty, this dividend strategy with $U_{t}>c$ at a point where $X_{t}^{\pi}=0$ will not be optimal. So we can assume without restriction that $\left\{Z_{t}\right\}$ only increases when the claim arrives; that is, it is a pure jump process. Thus

$$
\sum_{\substack{0 \leq s<\tau^{\pi} \\ X_{s}^{\pi} \neq X_{s+}^{\pi}}} e^{-\delta s}\left[V\left(X_{s+}^{\pi}\right)-V\left(X_{s}^{\pi}\right)\right]=\phi \int_{0}^{\tau^{\pi}} e^{-\delta s} \mathrm{~d} Z_{s} .
$$


When claim arrives, $X_{s-}^{\pi} \neq X_{s}^{\pi}$. Then

$$
\begin{aligned}
M\left(\tau^{\pi}\right)= & M\left(\sigma^{\pi} \wedge h\right) \\
= & \sum_{\substack{0 \leq \leq \leq \tau^{\pi} \\
X_{s-}^{\pi} \neq X_{s}^{\pi}}} e^{-\delta s}\left[V\left(X_{s}^{\pi}\right)-V\left(X_{s-}^{\pi}\right)\right]-\lambda \\
& \times \int_{0}^{\tau^{\pi}} \int_{0}^{\infty} e^{-\delta s}\left(V\left(X_{s-}^{\pi}-y\right)-V\left(X_{s-}^{\pi}\right)\right) \mathrm{d} F(y) \mathrm{d} s
\end{aligned}
$$

is a martingale with $M(0)=0$. So from the dynamic programming principle in (18), we have

$$
\begin{aligned}
& V(x) \\
& \geq E_{x}\left[\int_{0}^{\tau^{\pi}} e^{-\delta s} U_{s} \mathrm{~d} s-\phi \int_{0}^{\tau^{\pi}} e^{-\delta s} \mathrm{~d} Z_{s}+V(x)\right. \\
& +\int_{0}^{\tau^{\pi}} e^{-\delta s}\left[\left(c-U_{s}\right) V^{\prime}\left(X_{s-}^{\pi}\right)-\delta V\left(X_{s-}^{\pi}\right)\right. \\
& +\lambda \int_{0}^{\infty} V\left(X_{s-}^{\pi}-y\right) \\
& \left.\left.-V\left(X_{s-}^{\pi}\right) \mathrm{d} F(y)\right] \mathrm{d} s+\phi \int_{0}^{\tau^{\pi}} e^{-\delta s} \mathrm{~d} Z_{s}\right] .
\end{aligned}
$$

Equivalently

$$
\begin{aligned}
E_{x}\left[\int_{0}^{\tau^{\pi}} e^{-\delta s}[\right. & \left(c-U_{s}\right) V^{\prime}\left(X_{s-}^{\pi}\right)+U_{s} \\
& +\lambda \int_{0}^{\infty} V\left(X_{s-}^{\pi}-y\right) \mathrm{d} F(y) \\
& \left.\left.-(\lambda+\delta) V\left(X_{s-}^{\pi}\right)\right] \mathrm{d} s\right] \leq 0 .
\end{aligned}
$$

Dividing $E \tau^{\pi}$ in (23) and letting $h \rightarrow 0$ yield

$$
\begin{aligned}
(c-u) & V^{\prime}(x)+u \\
+ & \lambda \int_{0}^{\infty} V(x-y) \mathrm{d} F(y)-(\lambda+\delta) V(x) \leq 0 .
\end{aligned}
$$

We have proved that $V(x)$ is increasing, continuous, and nonnegative, so the above inequality can be rewritten as

$$
\begin{aligned}
(c-u) & V^{\prime}(x)+u \\
& +\lambda \int_{0}^{x+z} V(x-y) \mathrm{d} F(y)-(\lambda+\delta) V(x) \leq 0
\end{aligned}
$$

for $z \in R_{+}$.

On the other hand, consider a strategy by receiving $\varepsilon>0$ from the shareholder immediately and following the optimal strategy for the capital $x+\varepsilon$ afterwards; then $V(x) \geq V(x+$ $\varepsilon)-\phi \varepsilon$. Letting $\varepsilon \rightarrow 0$, we get

$$
V^{\prime}(x) \leq \phi .
$$

A more sophisticated analysis shows that one of the inequalities (25) and (26) is always tight (see Fleming and Soner [11]).

As a result, we get the following HJB equation satisfied by the value function $V(x)$ on $[0, \infty)$ :

$$
\begin{aligned}
\max \left\{\operatorname { s u p } _ { \substack { 0 \leq u \leq u _ { 0 } \\
z \in R _ { + } } } \left\{(c-u) V^{\prime}(x)+u+\lambda\right.\right. \\
\left.\quad \times \int_{0}^{x+z} V(x-y) \mathrm{d} F(y)-(\lambda+\delta) V(x)\right\}, \\
\left.V^{\prime}(x)-\phi\right\}=0 .
\end{aligned}
$$

The expressions to be maximized are

$$
u\left(1-V^{\prime}(x)\right), \quad \int_{0}^{x+z} V(x-y) \mathrm{d} F(y) .
$$

First, because $u\left(1-V^{\prime}(x)\right)$ is linear in $u, u^{*}(x)$ maximizing $u\left(1-V^{\prime}(x)\right)$ is

$$
u^{*}(x)= \begin{cases}0, & \text { if } V^{\prime}(x)>1 \\ u_{0}, & \text { if } V^{\prime}(x) \leq 1\end{cases}
$$

Second, we will maximize $\int_{0}^{x+z} V(x-y) \mathrm{d} F(y)$. Because $V(x) \geq 0$, we can define $z^{*}=-\inf \{z ; V(z)>0\}$. If $x<0$, the shareholders either inject capital to survive the company or default to do so. Ruin occurs in the latter case, while in the former case $V(x)$ will be linear when $x<0$; that is, $V(x)=V(0)+\phi x$. Thus, from the definition of $z^{*}$, we have

$$
z^{*}=\frac{V(0)}{\phi} .
$$

In fact, $z^{*}$ is the maximal deficit that the shareholder should bare. We call $-z^{*}$ the optimal lower capital injection barrier.

If $V(x)$ is concave on $(0, \infty)$, then there exists an optimal dividend barrier $b:=\inf \left\{x: V^{\prime}(x) \leq 1\right\}$ with

$$
u^{*}(x)= \begin{cases}0, & \text { if } x<b \Longleftrightarrow V^{\prime}(x)>1 \\ u_{0}, & \text { if } x \geq b \Longleftrightarrow V^{\prime}(x) \leq 1 .\end{cases}
$$

And also a barrier $a_{0}:=\sup \left\{x, V^{\prime}(x) \geq \phi\right\}$. If the reserves become less than $a_{0}$, according to $z^{*}$, the shareholders may take actions between the following two choices.

(a) If the deficit is larger than $z^{*}$, they refuse to inject any capital and ruin occurs.

(b) Otherwise, they inject capital and the injected amount should recover the reserves to $a_{0}$. If $a_{0}<0$, the injected amount could not survive the company. Therefore, we define the optimal upper capital injection barrier as $a=a_{0} \vee 0$. 
Recall that in the literature (e.g., Kulenko and Schmidli [9] and He and Liang [4, 5]) concerning the capital injection strategy, considering the discounting, it can not be optimal to inject capital before they really are necessary. Therefore, the shareholders postpone injecting capital as long as possible and just conjecture that it is optimal to do so only when the reserves become 0 . In the next proposition, from the mathematical point of view, we will give a rigorous proof of $a=0$.

Proposition 3. If $V(x)$ is concave on $(0, \infty)$, the optimal upper capital injection barrier $a=0$.

Proof. Under the assumption, $a$ is unique. Suppose $a>0$. So $V(x)=V(0)+\phi x$ when $x \in\left[-z^{*}, a\right]$. Note that $V^{\prime}(a)=$ $V^{\prime}(0)=\phi \cdot V(x)$ fulfils the HJB equation (27), so at $x=0$

$$
c \phi+\lambda \int_{0}^{V(0) / \phi}[V(0)-\phi y] \mathrm{d} F(y)-(\lambda+\delta) V(0) \leq 0 .
$$

If we take $V(0)=V(a)-\phi a$ into the left side of (32), the expression turns into

$$
\begin{aligned}
c \phi+ & \lambda \int_{0}^{V(0) / \phi}[V(a)-\phi a-\phi y] \mathrm{d} F(y) \\
& -(\lambda+\delta)[V(a)-\phi a] .
\end{aligned}
$$

At the optimal upper capital injection barrier $a$,

$$
c V^{\prime}(a+)+\lambda \int_{0}^{V(a) / \phi}[V(a)-\phi y] \mathrm{d} F(y)-(\lambda+\delta) V(a)=0 .
$$

It implies

$$
(\lambda+\delta) V(a)=c V^{\prime}(a+)+\lambda \int_{0}^{V(a) / \phi}[V(a)-\phi y] \mathrm{d} F(y) .
$$

Pulling (35) into (33), we can rewrite the expression by

$$
\begin{aligned}
c \phi+ & \lambda \int_{0}^{V(0) / \phi}[V(a)-\phi a-\phi y] \mathrm{d} F(y)+(\lambda+\delta) \phi a \\
& -c V^{\prime}(a+)-\lambda \int_{0}^{V(a) / \phi}[V(a)-\phi y] \mathrm{d} F(y) \\
= & -\lambda \int_{0}^{V(0) / \phi} \phi a \mathrm{~d} F(y)-\lambda \int_{V(0) / \phi}^{V(a) / \phi}[V(a)-\phi y] \mathrm{d} F(y) \\
& +(\lambda+\delta) \phi a+c\left(\phi-V^{\prime}(a+)\right) \\
= & -\lambda \phi a F\left(\frac{V(0)}{\phi}\right)+\lambda V(a) F\left(\frac{V(0)}{\phi}\right) \\
& -\lambda V(a) F\left(\frac{V(a)}{\phi}\right)+\phi \lambda \int_{e}^{V(a) / \phi} y \mathrm{~d} F(y)
\end{aligned}
$$

$$
\begin{aligned}
& +(\lambda+\delta) \phi a+c\left(\phi-V^{\prime}(a+)\right) \\
= & -\lambda \phi a F\left(\frac{V(0)}{\phi}\right)+\lambda V(a) F\left(\frac{V(0)}{\phi}\right) \\
& -\lambda V(a) F\left(\frac{V(a)}{\phi}\right)+c\left(\phi-V^{\prime}(a+)\right) \\
& -\lambda V(0) F\left(\frac{V(0)}{\phi}\right)+\lambda V(a) F\left(\frac{V(a)}{\phi}\right) \\
& -\lambda \phi \int_{V(0) / \phi}^{V(a) / \phi} F(y) \mathrm{d} y+(\lambda+\delta) \phi a \\
= & {[-\lambda \phi a+\lambda V(a)-\lambda \phi V(0)] F\left(\frac{V(a)}{\phi}\right)+(\lambda+\delta) \phi a } \\
& -\lambda \phi \int_{V(0) / \phi}^{V(a) / \phi} F(y) \mathrm{d} y+c\left(\phi-V^{\prime}(a+)\right) \\
= & (\lambda+\delta) \phi a-\lambda \phi \int_{V(0) / \phi}^{V(a) / \phi} F(y) \mathrm{d} y+c\left(\phi-V^{\prime}(a+)\right) .
\end{aligned}
$$

However

$$
\begin{aligned}
-\lambda \phi & \int_{V(0) / \phi}^{V(a) / \phi} F(y) \mathrm{d} y+(\lambda+\delta) \phi a+c\left(\phi-V^{\prime}(a+)\right) \\
& \geq-\lambda V(a)+\lambda V(0)+(\lambda+\delta) \phi a \\
& =-\lambda(V(a)-V(0)-\phi a)+\delta \phi a \\
& =\delta \phi a>0,
\end{aligned}
$$

which is contradictory with (32). So $a>0$ is impossible and $a=0$ is proved.

The above proposition tells us that the moment when deficit occurs is just the time the shareholders consider to inject capital.

Proposition 4. If $V(x)$ is concave on $(0, \infty)$, it is continuously differentiable on $(0, \infty)$.

Proof. From the concavity of $V(x),(31)$ is true. When $x \in$ $(0, b)$, from HJB equation $(27)$, and $V(x)$ is Lipschitz continuous, so

$$
\begin{aligned}
& c V^{\prime}(x+)-(\lambda+\delta) V(x)+\lambda \int_{0}^{V(0) / \phi} V(x-y) \mathrm{d} F(y) \\
& =c V^{\prime}(x-)-(\lambda+\delta) V(x) \\
& \quad+\lambda \int_{0}^{V(0) / \phi} V(x-y) \mathrm{d} F(y)=0 .
\end{aligned}
$$


Thus $V^{\prime}(x-)=V^{\prime}(x+)$. Similarly, we can proof $V(x)$ is continuously differentiable on $(b, \infty)$. Now suppose $b>0$. Note

$$
\begin{gathered}
\left(c-u_{0}\right) V^{\prime}(b+)+u_{0}-(\lambda+\delta) V(b) \\
+\lambda \int_{0}^{V(0) / \phi} V(b-y) \mathrm{d} F(y)=0 \\
c V^{\prime}(b-)-(\lambda+\delta) V(b)+\lambda \int_{0}^{V(0) / \phi} V(b-y) \mathrm{d} F(y)=0 .
\end{gathered}
$$

So $c V^{\prime}(b-)=u_{0}+\left(c-u_{0}\right) V^{\prime}(b+)$ or equivalently $c\left(V^{\prime}(b-)-\right.$ $\left.V^{\prime}(b+)\right)=u_{0}\left(1-V^{\prime}(b+)\right)$.

If $u_{0}<c$, either $V^{\prime}(b-)=V^{\prime}(b+)=1$ or $1>V^{\prime}(b-)$. The latter is impossible, so $V(x)$ is continuously differentiable under this case.

If $u_{0} \geq c$, the reserve stays at $b$ until the first claim occurs because dividend is a barrier strategy. $b$ is independent of the constant $u_{0}$. In fact, because the process does not leave the interval $[0, b]$ and the corresponding strategy is admissible for any $u_{0} \geq c$, it must be optimal for any initial value in $[0, b]$. For $x=b$, the expected discounted dividends until the first claim are

$$
\lambda \int_{0}^{\infty} e^{-\lambda t} \int_{0}^{t} c e^{-\delta s} \mathrm{~d} s \mathrm{~d} t=\frac{\lambda c}{\delta} \int_{0}^{\infty}\left(1-e^{-\delta t}\right) e^{-\lambda t} \mathrm{~d} t=\frac{c}{\lambda+\delta} .
$$

The expected discounted dividends after the first claim are

$$
\begin{gathered}
\lambda \int_{0}^{\infty} e^{-\lambda t} \int_{0}^{b} e^{-\delta t} V(b-y) \mathrm{d} F(y) \mathrm{d} t \\
\quad+\lambda \int_{0}^{\infty} e^{-\lambda t} \int_{b}^{b+z^{*}} e^{-\delta t}[V(0)+\phi(b-y)] \mathrm{d} F(y) \mathrm{d} t \\
=\frac{\lambda}{\lambda+\delta}\left[\int_{0}^{b} V(b-y) \mathrm{d} F(y)\right. \\
\left.\quad+\int_{b}^{b+z^{*}}[V(0)+\phi(b-y)] \mathrm{d} F(y)\right] .
\end{gathered}
$$

Hence, the value at $b$ can be characterized as

$$
\begin{aligned}
V(b)=\frac{c}{\lambda+\delta}+\frac{\lambda}{\lambda+\delta}[ & \int_{0}^{b} V(b-y) \mathrm{d} F(y) \\
& \left.+\int_{b}^{b+z^{*}}[V(0)+\phi(b-y)] \mathrm{d} F(y)\right] .
\end{aligned}
$$

Pulling $V(b)$ into (39), we find $V^{\prime}(b-)=V^{\prime}(b+)=1$. So $V(x)$ is continuously differentiable in this case, too.

It holds in an interval $\left(T_{i-1}, T_{i}\right)$ between two claims that $\mathrm{d} X_{t}^{\pi}=\left(c-U_{t}\right) \mathrm{d} t . \Delta Z_{T_{i}}=Z_{T_{i}+}-Z_{T_{i}}$ denotes the injected capital at the $i$ th claim arrivals. (i) If $X_{T_{i^{-}}}^{\pi}-Y_{i} \geq 0$, then $\Delta Z_{T_{i}}=0$;

(ii) If $-z^{*}<X_{T_{i}-}^{\pi}-Y_{i}<0$, then the shareholders pay as much that $X_{T_{i}+}^{\pi}=X_{T_{i}-}^{\pi}-Y_{i}+\Delta Z_{T_{i}}=0$. That is, $\Delta Z_{T_{i}}=0-\left(X_{T_{i}-}^{\pi}-Y_{i}\right)$. In this case, the value function fulfils

$$
\begin{array}{r}
V\left(X_{T_{i^{+}}}^{\pi}\right)(=V(0))=V\left(X_{T_{i^{-}}}^{\pi}-Y_{i}\right)+\phi \Delta Z_{T_{i}}, \\
\text { if }-z^{*}<X_{T_{i^{-}}}^{\pi}-Y_{i}<0 .
\end{array}
$$

(iii) If $X_{T_{i}{ }^{-}}^{\pi}-Y_{i} \leq-z^{*}$, then the shareholders would get a negative net profit as long as they cover the deficit (because $\left.V(0)-\phi \Delta Z_{T_{i}}<0\right)$. It is unreasonable. Hence, they prefer to "no-injection-no-profit" and refuse to inject capital anymore. In this case, bankruptcy occurs and $T^{\pi}=T_{i}$. So

$$
\begin{aligned}
V\left(X_{T_{i}+}^{\pi}\right)=V\left(X_{T_{i}}^{\pi}\right)= & V\left(X_{T_{i^{-}}}^{\pi}-Y_{i}\right)=0, \\
& \text { if } X_{T_{i^{-}}}^{\pi}-Y_{i} \leq-z^{*} .
\end{aligned}
$$

Based on the discussion above, when $x<0$, we can express $V(x)$ by

$$
V(x)= \begin{cases}0, & \text { if } x \leq-z^{*}, \\ V(0)+\phi x, & \text { if }-z^{*}<x<0 .\end{cases}
$$

Thus it suffices to consider solutions $f$ to the HJB equation with the properties

$$
\begin{gathered}
f(x)=0, \quad \text { if } x \leq-\frac{f(0)}{\phi} . \\
f(x)=f(0)+\phi x, \quad \text { if }-\frac{f(0)}{\phi}<x<0 .
\end{gathered}
$$

Lemma 5. Let $f(x)$ be an increasing, bounded, and nonnegative solution to (27) with properties (46) and (47). Then for any admissible strategy $\pi \in \Pi^{r}$, the process

$$
\begin{aligned}
& \left\{f\left(X_{t \wedge T^{\pi}}^{\pi}\right) e^{-\delta\left(t \wedge T^{\pi}\right)}-f(x)-\phi \int_{0}^{t \wedge T^{\pi}} e^{-\delta s} d Z_{s}\right. \\
& -\int_{0}^{t \wedge T^{\pi}}\left[\left(c-U_{s}\right) f^{\prime}\left(X_{s}^{\pi}\right)-(\lambda+\delta) f\left(X_{s}^{\pi}\right)\right. \\
& \left.\left.+\lambda \int_{0}^{X_{s}^{\pi}+(f(0) / \phi)} f\left(X_{s}^{\pi}-y\right) d F(y)\right] e^{-\delta s} d s\right\}
\end{aligned}
$$

is a martingale.

Proof. First we decompose $f\left(X_{t \wedge T^{\pi}}^{\pi}\right) e^{-\delta\left(t \wedge T^{\pi}\right)}$

$$
\begin{aligned}
f & \left(X_{t \wedge T^{\pi}}^{\pi}\right) e^{-\delta\left(t \wedge T^{\pi}\right)} \\
& =f\left(X_{0+}^{\pi}\right)+\sum_{i=1}^{N_{t \wedge T^{\pi}}}\left[f\left(X_{T_{i}+}^{\pi}\right) e^{-\delta T_{i}}-f\left(X_{T_{i-1}+}^{\pi}\right) e^{-\delta T_{i-1}}\right]
\end{aligned}
$$




$$
\begin{aligned}
& +f\left(X_{t \wedge T^{\pi}}^{\pi}\right) e^{-\delta\left(t \wedge T^{\pi}\right)}-f\left(X_{T_{N_{t \wedge T^{\pi}}}^{\pi}}^{\pi}\right) e^{-\delta T_{N_{t \wedge T^{\pi}}}} \\
& =f\left(X_{0+}^{\pi}\right)+\sum_{i=1}^{N_{t \wedge N^{\pi}} \pi}\left[f\left(X_{T_{i}+}^{\pi}\right)-f\left(X_{T_{i}-}^{\pi}\right)\right] e^{-\delta T_{i}} \\
& +\sum_{i=1}^{N_{t \wedge \pi^{\pi}}}\left[f\left(X_{T_{i}-}^{\pi}\right) e^{-\delta T_{i}}-f\left(X_{T_{i-1}+}^{\pi}\right) e^{-\delta T_{i-1}}\right] \\
& +f\left(X_{t \wedge T^{\pi}}^{\pi}\right) e^{-\delta\left(t \wedge T^{\pi}\right)}-f\left(X_{T_{N_{t \wedge T^{\pi}}}^{\pi}}^{\pi}\right) e^{-\delta T_{N_{t \wedge T^{\pi}}}} \\
& =f(x)+\phi \Delta Z_{T_{0}}+\sum_{i=1}^{N_{t \wedge T^{\pi}}}\left[f\left(X_{T_{i}-}^{\pi}-Y_{i}\right)-f\left(X_{T_{i}-}^{\pi}\right)\right] e^{-\delta T_{i}} \\
& +\phi \sum_{i=1}^{N_{t \wedge \pi^{\pi}}} \Delta Z_{T_{i}} e^{-\delta T_{i}}+\sum_{i=1}^{N_{t \wedge T^{\pi}}} \int_{T_{i-1}+}^{T_{i}-} d e^{-\delta s} f\left(X_{s}^{\pi}\right) \\
& +\int_{T_{N_{t \wedge T^{\pi}}}}^{t \wedge T^{\pi}} \mathrm{d} e^{-\delta s} f\left(X_{s}^{\pi}\right) \\
& =f(x)+\sum_{i=1}^{N_{t \wedge T^{\pi}}}\left[f\left(X_{T_{i^{-}}}^{\pi}-Y_{i}\right)-f\left(X_{T_{i^{-}}}^{\pi}\right)\right] e^{-\delta T_{i}} \\
& +\int_{T_{N_{t \wedge T^{\pi}}}}^{t \wedge T^{\pi}}\left[\left(c-U_{s}\right) f^{\prime}\left(X_{s}^{\pi}\right)-\delta f\left(X_{s}^{\pi}\right)\right] e^{-\delta s} \mathrm{~d} s \\
& +\sum_{i=1}^{N_{t \wedge N^{\pi}} \pi} \int_{T_{i-1}+}^{T_{i}-}\left[\left(c-U_{s}\right) f^{\prime}\left(X_{s}^{\pi}\right)-\delta f\left(X_{s}^{\pi}\right)\right] e^{-\delta s} \mathrm{~d} s \\
& +\phi \int_{0}^{t \wedge T^{\pi}} e^{-\delta s} \mathrm{~d} Z_{s} \\
& =f(x)+\sum_{i=1}^{N_{t \wedge T^{\pi}}}\left[f\left(X_{T_{i^{-}}}^{\pi}-Y_{i}\right)-f\left(X_{T_{i^{-}}}^{\pi}\right)\right] e^{-\delta T_{i}} \\
& +\phi \int_{0}^{t \wedge T^{\pi}} e^{-\delta s} \mathrm{~d} Z_{s} \\
& +\int_{0}^{t \wedge T^{\pi}}\left[\left(c-U_{s}\right) f^{\prime}\left(X_{s}^{\pi}\right)-\delta f\left(X_{s}^{\pi}\right)\right] e^{-\delta s} \mathrm{~d} s .
\end{aligned}
$$

Then in order to make the process $\left\{\sum_{i=1}^{N_{t \wedge T^{\pi}}}\left[f\left(X_{T_{i}{ }^{-}}^{\pi}-Y_{i}\right)-\right.\right.$ $\left.\left.f\left(X_{T_{i}-}^{\pi}\right)\right] e^{-\delta T_{i}}-\int_{0}^{t \wedge T^{\pi}} g\left(X_{s}^{\pi}\right) \mathrm{d} s\right\}$ become a martingale with the expected value 0 , we must find a measurable function $g$. Since the above expression can be written as

$$
\begin{aligned}
\sum_{i=1}^{N_{t \wedge \Lambda^{\pi}}}\{ & {\left.\left[f\left(X_{T_{i}-}^{\pi}-Y_{i}\right)-f\left(X_{T_{i}-}^{\pi}\right)\right] e^{-\delta T_{i}}-\int_{T_{i-1}}^{T_{i}} g\left(X_{s}^{\pi}\right) \mathrm{d} s\right\} } \\
& -\int_{T_{N_{t \wedge T^{\pi}}}^{t}}^{t} g\left(X_{s}^{\pi}\right) \mathrm{d} s
\end{aligned}
$$

it is enough to replace $t$ by $T_{1} \wedge t$; that is,

$$
\left[f\left(X_{T_{1}-}^{\pi}-Y_{1}\right)-f\left(X_{T_{1}-}^{\pi}\right)\right] e^{-\delta T_{1}} 1_{\left(T_{1} \leq t\right)}-\int_{0}^{t \wedge T_{1}} g\left(X_{s}^{\pi}\right) \mathrm{d} s .
$$

Because the exponential distribution is lack of memory, we only consider the expected value. $g$ will satisfy

$$
\begin{gathered}
E\left\{\left[f\left(X_{T_{1}-}^{\pi}-Y_{1}\right)-f\left(X_{T_{1}-}^{\pi}\right)\right] e^{-\delta T_{1}} 1_{\left(T_{1} \leq t\right)}\right. \\
\left.-\int_{0}^{t \wedge T_{1}} g\left(X_{s}^{\pi}\right) \mathrm{d} s\right\}=0 .
\end{gathered}
$$

The expected values of the first and the second part are

$$
\begin{gathered}
\int_{0}^{t} \lambda e^{-\lambda s} e^{-\delta s}\left\{\int_{0}^{x+\int_{0}^{s}\left(c-U_{v}\right) \mathrm{d} v+f(0) / \phi} f\right. \\
\quad \times\left(x+\int_{0}^{s}\left(c-U_{v}\right) \mathrm{d} v-y\right) \mathrm{d} F(y) \\
\left.\quad-f\left(x+\int_{0}^{s}\left(c-U_{v}\right) \mathrm{d} v\right)\right\} \mathrm{d} s \\
\int_{0}^{t} \lambda e^{-\lambda s} \int_{0}^{s} g\left(x+\int_{0}^{v}\left(c-U_{w}\right) \mathrm{d} w\right) \mathrm{d} v \mathrm{~d} s \\
+e^{-\lambda t} \int_{0}^{t} g\left(x+\int_{0}^{s}\left(c-U_{v}\right) \mathrm{d} v\right) \mathrm{d} s \\
=\int_{0}^{t} e^{-\lambda s} g\left(x+\int_{0}^{s}\left(c-U_{v}\right) \mathrm{d} v\right) \mathrm{d} s .
\end{gathered}
$$

Thus we can choose

$$
\begin{aligned}
g\left(X_{t}^{\pi}\right) & =\lambda e^{-\delta t}\left(\int_{0}^{X_{t}^{\pi}+f(0) / \phi} f\left(X_{t}^{\pi}-y\right) \mathrm{d} F(y)-f\left(X_{t}^{\pi}\right)\right) \\
& =\lambda e^{-\delta t} \int_{0}^{X_{t}^{\pi}+f(0) / \phi} f\left(X_{t}^{\pi}-y\right) \mathrm{d} F(y)-\lambda e^{-\delta t} f\left(X_{t}^{\pi}\right) .
\end{aligned}
$$

So

$$
\begin{aligned}
& \left\{\sum_{i=1}^{N_{t \wedge T^{\pi}}}\left[f\left(X_{T_{i^{-}}}^{\pi}-Y_{i}\right)-f\left(X_{T_{i^{-}}}^{\pi}\right)\right] e^{-\delta T_{i}}-\lambda\right. \\
& \left.\quad \times \int_{0}^{t \wedge T^{\pi}} e^{-\delta s}\left[\int_{0}^{X_{s}^{\pi}+f(0) / \phi} f\left(X_{s}^{\pi}-y\right) \mathrm{d} F(y)-f\left(X_{s}^{\pi}\right)\right] \mathrm{d} s\right\}
\end{aligned}
$$

and, also, the process

$$
\begin{aligned}
& \left\{f\left(X_{t \wedge T^{\pi}}^{\pi}\right) e^{-\delta\left(t \wedge T^{\pi}\right)}-f(x)-\phi \int_{0}^{t \wedge T^{\pi}} e^{-\delta s} \mathrm{~d} Z_{s}\right. \\
& -\int_{0}^{t \wedge T^{\pi}}\left[\left(c-U_{s}\right) f^{\prime}\left(X_{s}^{\pi}\right)-(\lambda+\delta) f\left(X_{s}^{\pi}\right)\right. \\
& \left.\left.\quad+\lambda \int_{0}^{X_{s}^{\pi}+f(0) / \phi} f\left(X_{s}^{\pi}-y\right) \mathrm{d} F(y)\right] e^{-\delta s} \mathrm{~d} s\right\}
\end{aligned}
$$

are $\left\{\mathscr{F}_{t}\right\}$-martingales with expected value 0 .

The following theorem serves as a verification theorem. 
Theorem 6. Let $f(x)$ be an increasing and bounded solution to (27) with the properties (46) and (47). Then $\lim _{x \rightarrow \infty} f(x)=$ $u_{0} / \delta$ and $f(x)=V(x)$ on $R_{+}$. The optimal capital injection and dividend barriers are given by (30) and (31).

Proof. Because $f(x)$ is increasing and bounded, we assume $\lim _{x \rightarrow \infty} f(x)=f_{0}$. Then there exists a sequence $x_{n} \rightarrow \infty$ such that $f^{\prime}\left(x_{n}\right) \rightarrow 0$. Let $u_{n}=u\left(x_{n}\right)$. From the definition of the optimal dividend strategy, we can assume that $u_{n}=u_{0}$. As $n \rightarrow \infty$, the first term in (27) turns to

$$
\begin{aligned}
0 & =\left(c-u_{0}\right) f^{\prime}\left(x_{n}\right)+u_{0}-\delta f\left(x_{n}\right) \\
& +\lambda\left[\int_{0}^{x_{n}+f(0) / \phi} f\left(x_{n}-y\right) \mathrm{d} F(y)-f\left(x_{n}\right)\right] \\
& \longrightarrow-\delta f_{0}+u_{0} .
\end{aligned}
$$

Equivalently we have $\lim _{x \rightarrow \infty} f(x)=u_{0} / \delta$.

Let $T^{*}$ be the ruin time under the strategies (30) and (31) and $V^{*}(x)$ the corresponding value. From Lemma 5 and the HJB equation (27), we have

$$
\begin{aligned}
& \left\{f\left(X_{t \wedge T^{*}}^{\pi^{*}}\right) e^{-\delta\left(t \wedge T^{*}\right)}-f(x)\right. \\
& \left.+\int_{0}^{t \wedge T^{*}} e^{-\delta s} U_{s}^{*} \mathrm{~d} s-\phi \int_{0}^{t \wedge T^{*}} e^{-\delta s} \mathrm{~d} Z_{s}^{*}\right\}
\end{aligned}
$$

is a martingale with expected value 0 . Then

$$
\begin{gathered}
f(x)=E_{x}\left[f\left(X_{t \wedge T^{*}}^{\pi^{*}}\right) e^{-\delta\left(t \wedge T^{*}\right)}+\int_{0}^{t \wedge T^{*}} e^{-\delta s} U_{s}^{*} \mathrm{~d} s\right. \\
\left.-\phi \int_{0}^{t \wedge T^{*}} e^{-\delta s} \mathrm{~d} Z_{s}^{*}\right] .
\end{gathered}
$$

Since $f$ is bounded and from the bounded convergence theorem, as $t \rightarrow \infty$, we get that $E\left[f\left(X_{t \wedge T^{*}}^{\pi^{*}}\right) e^{-\delta\left(t \wedge T^{*}\right)}\right] \rightarrow$ 0 . The other terms are monotone, when $t \rightarrow \infty$, by interchanging the limit and integration, so we obtain $f(x)=$ $V^{*}(x) \leq V(x)$.

On the other hand, because $f(x)$ is increasing and satisfies (46), $f(x)$ is nonnegative on $(-\infty, \infty)$. For any admissible strategy $\pi$, HJB equation (27) gives that

$$
\begin{array}{r}
f(x) \geq E_{x}\left[f\left(X_{t \wedge T^{\pi}}^{\pi}\right) e^{-\delta\left(t \wedge T^{\pi}\right)}+\int_{0}^{t \wedge T^{\pi}} e^{-\delta s} U_{s} \mathrm{~d} s\right. \\
\left.-\phi \int_{0}^{t \wedge T^{\pi}} e^{-\delta s} \mathrm{~d} Z_{s}\right] \\
\geq E_{x}\left[\int_{0}^{t \wedge T^{\pi}} e^{-\delta s} U_{s} \mathrm{~d} s-\phi \int_{0}^{t \wedge T^{\pi}} e^{-\delta s} \mathrm{~d} Z_{s}\right] .
\end{array}
$$

Let $t \rightarrow \infty$; then $f(x) \geq V^{\pi}(x)$, which means $f(x) \geq V(x)$. Thus, $f(x)=V(x)$.

Based on the discussion above, if $V(x)$ is concave on $(0, \infty)$, it is optimal for the shareholders to take no action as long as the reserve process takes value in $(0, b)$. When the process reaches or exceeds the barrier $b$, dividends have to be paid at the maximal rate $u_{0}$. When the reserve is less than 0 , the shareholders should consider either to inject capital to recover the reserve to 0 or default to do so. If the decifit is less than $z^{*}$, the shareholders can earn positive net profit. So they inject capital which covers the deficit to survive the company. Otherwise, once the deficit is larger than $z^{*}$, the shareholders refuse to do so and ruin occurs.

Remark 7. Diffusion models can be used to approximate the Cramér-Lundberg risk model. During the recent decades, they have been applied to insurance modeling setting extensively. See Radner and Shepp [12], Asmussen and Taksar [13], and Højgaard and Taksar [14, 15], Sethi and Taksar [2], and so forth. Diffusion models have the advantage that some very explicit optimal controls and a smooth value function can be made. Hopefully, these can help to take almost optimal strategies for the original risk model. However, this statement is not trivial.

The optimal dividend and issuance equity strategies (or combined with other strategies) in diffusion risk model had been studied by Løkka and Zervos [3], He and Liang [4, 5], and so forth. In their paper, depending on the relationships between the coefficients, it is optimal for the company either to involve no issuance equity or to involve issuance equity without ruin. In this paper, our conclusion in the CramérLundberg risk model is that the optimal capital injection strategy will depend on the deficit. Once the deficit is large, ruin will still occur. Thus the optimal capital injection strategy looks different for these two models and the diffusion approximations are not effective here.

Discussion on whether the diffusion approximation is true can be found in Maglaras [16] and Bäuerle [6], and so forth.

\section{Unrestricted Dividends}

In this section, we will discuss the dividend strategy without restriction. Here all increasing, adapted, and càdlàg processes are allowed to be the dividend strategy. Let $\Pi$ denote the set of all admissible strategies. The value of an admissible strategy $\pi$ is

$$
V^{\pi}(x)=E\left[\int_{0-}^{T^{\pi}-} e^{-\delta t} \mathrm{~d} D_{t}-\phi \int_{0}^{T^{\pi}} e^{-\delta t} \mathrm{~d} Z_{t}\right] .
$$

The value function is $V(x)=\sup _{\pi \in \Pi} V^{\pi}(x)$.

Lemma 8. On $[0, \infty)$, the function $V(x)$ is increasing and local Lipschitz continuous; $V(x)-V(y) \geq x-y$ if $x \geq y$; $0 \leq V(x) \leq x+c / \delta$.

Proof. For any $\varepsilon>0$, define a strategy $\pi$ satisfing $V^{\pi}(y) \geq$ $V(y)-\varepsilon \cdot \pi^{\prime}$ is a new strategy for $x \geq y \cdot\left\{Z_{t}^{\prime}\right\}$ in $\pi^{\prime}$ is the same as $\left\{Z_{t}\right\}$ in $\pi$. While $\left\{D_{t}^{\prime}\right\}$ is defined as: $x-y$ is paid immediately as dividend and then the strategy $\left\{D_{t}\right\}$ with initial capital $y$ is followed. Therefore, $V(x) \geq x-y+V^{\pi}(y) \geq x-y+V(y)-\varepsilon$. From the arbitrary property of $\varepsilon$, we have $V(x)-V(y) \geq x-y$. In particulars, $V(x)$ is strictly increasing. 
Consider such a strategy $\pi$ : the initial capital $x$ is paid to the shareholders as dividends immediately and capital injection is forbidden. Then $V(x) \geq V^{\pi}(x) \geq 0$.

To get the upper bound of $V(x)$, we consider a strategy $\pi$. $\left\{D_{t}\right\}$ is defined as: if the initial capital is $x(x \geq 0)$, then $x$ is paid immediately and then the dividends are paid at rate $c$. If we donot take the capital injection into account, then $x+E_{x}\left[\int_{0}^{\infty} e^{-\delta t} c \mathrm{~d} t\right]=x+c / \delta$ is the upper bound of any admissible strategy $\pi$; that is, $V(x) \leq x+c / \delta$.

The local Lipschitz continuity follows by the local boundedness of $V(x)$ as in the proof of Lemma 2.

3.1. HJB Equation and the Optimal Strategies. Similar to the discussion in Section 2.2, $V(x)$ satisfies the following dynamic programming principle:

$$
\begin{gathered}
V(x)=\sup _{\pi} E_{x}\left[\int_{0-}^{\tau \wedge T^{\pi}} e^{-\delta t} \mathrm{~d} D_{t}-\phi \int_{0}^{\tau \wedge T^{\pi}} e^{-\delta t} \mathrm{~d} Z_{t}\right. \\
\left.+e^{-\delta\left(\tau \wedge T^{\pi}\right)} V\left(X_{\tau \wedge T^{\pi}}^{\pi}\right)\right]
\end{gathered}
$$

for $x \in R_{+}$and any $\left\{\mathscr{F}_{t}\right\}$-stopping time $\tau$.

For $x \geq 0$, similarly we define $\tau^{\pi}$ as in Section 2.2. Note that $\sigma^{\pi}=T^{\pi}$ is possible here. Applying Itô formula into $e^{-\delta \tau^{\pi}} V\left(X_{\tau^{\pi}}^{\pi}\right)$, we have

$$
\begin{aligned}
e^{-\delta \tau^{\pi}} V\left(X_{\tau^{\pi}}^{\pi}\right)= & V\left(X_{0-}^{\pi}\right) \\
& +\int_{0}^{\tau^{\pi}} e^{-\delta s} c V^{\prime}\left(X_{s-}^{\pi}\right)-\delta e^{-\delta s} V\left(X_{s-}^{\pi}\right) \mathrm{d} s \\
& +\sum_{\substack{0 \leq s \leq \tau^{\pi} \\
X_{s-}^{s} \neq X_{s}^{\pi}}} e^{-\delta s}\left[V\left(X_{s}^{\pi}\right)-V\left(X_{s-}^{\pi}\right)\right] \\
& +\sum_{\substack{0 \leq s<\tau^{\pi} \\
X_{s}^{\pi} \neq X_{s+}^{\pi}}} e^{-\delta s}\left[V\left(X_{s+}^{\pi}\right)-V\left(X_{s}^{\pi}\right)\right] .
\end{aligned}
$$

$X_{s}^{\pi} \neq X_{s+}^{\pi}$ only when capital is injected, so

$$
\sum_{\substack{0 \leq s<\tau^{\pi} \\ X_{s}^{\pi} \neq X_{s+}^{\pi}}} e^{-\delta s}\left[V\left(X_{s+}^{\pi}\right)-V\left(X_{s}^{\pi}\right)\right]=\phi \int_{0}^{\tau^{\pi}} e^{-\delta s} \mathrm{~d} Z_{s} .
$$

When claim arrives or dividend occurs, $X_{s^{-}}^{\pi} \neq X_{s}^{\pi}$. The jumps caused by claim arrivals lead to

$$
\begin{aligned}
M\left(\tau^{\pi}\right)= & M\left(\sigma^{\pi} \wedge h\right) \\
= & \sum_{\substack{0 \leq s \leq \tau^{\pi} \\
X_{s-}^{\pi} \neq X_{s}^{\pi}}} e^{-\delta s}\left[V\left(X_{s}^{\pi}\right)-V\left(X_{s^{-}}^{\pi}\right)\right] \\
& -\lambda \int_{0}^{\tau^{\pi}} \int_{0}^{\infty} e^{-\delta s}\left(V\left(X_{s^{-}}^{\pi}-y\right)-V\left(X_{s^{-}}^{\pi}\right)\right) \mathrm{d} F(y) \mathrm{d} s
\end{aligned}
$$

is a martingale with $M(0)=0$. And the amount of the aggregated jumps caused by dividend are $-\int_{0-}^{\tau^{\pi}} e^{-\delta s} \mathrm{~d} D_{s}$. So from the dynamic programming principle (62), yields

$V(x)$

$$
\begin{aligned}
& \geq E_{x}\left[\int_{0-}^{\tau^{\pi}} e^{-\delta s} \mathrm{~d} D_{s}-\phi \int_{0}^{\tau^{\pi}} e^{-\delta s} \mathrm{~d} Z_{s}+V(x)\right. \\
& +\int_{0}^{\tau^{\pi}} e^{-\delta s}\left[c V^{\prime}\left(X_{s-}^{\pi}\right)-\delta V\left(X_{s-}^{\pi}\right)+\lambda\right. \\
& \left.\quad \times \int_{0}^{\infty} V\left(X_{s-}^{\pi}-y\right)-V\left(X_{s-}^{\pi}\right) \mathrm{d} F(y)\right] \mathrm{d} s \\
& \left.\quad-\int_{0-}^{\tau^{\pi}} e^{-\delta s} \mathrm{~d} D_{s}+\phi \int_{0}^{\tau^{\pi}} e^{-\delta s} \mathrm{~d} Z_{s}\right] .
\end{aligned}
$$

Equivalently

$$
\begin{gathered}
E_{x}\left[\int _ { 0 } ^ { \tau ^ { \pi } } e ^ { - \delta s } \left[c V^{\prime}\left(X_{s-}^{\pi}\right)+\lambda \int_{0}^{\infty} V\left(X_{s-}^{\pi}-y\right) \mathrm{d} F(y)\right.\right. \\
\left.\left.-(\lambda+\delta) V\left(X_{s-}^{\pi}\right)\right] \mathrm{d} s\right] \leq 0 .
\end{gathered}
$$

If $T^{\pi}=0$, then $\tau^{\pi}=0$. Therefore (67) gives no information. If $T^{\pi}>0$, we can choose $\varepsilon$ such that $E \tau^{\pi}>0$. Dividing $E \tau^{\pi}$ in (67) and letting $h \rightarrow 0$, so

$$
c V^{\prime}(x)+\lambda \int_{0}^{\infty} V(x-y) \mathrm{d} F(y)-(\lambda+\delta) V(x) \leq 0 .
$$

Also we can rewrite the above inequality by

$$
c V^{\prime}(x)+\lambda \int_{0}^{x+z} V(x-y) \mathrm{d} F(y)-(\lambda+\delta) V(x) \leq 0
$$

for $z \in R_{+}$.

Refering to the proof of (26), we have

$$
V^{\prime}(x) \leq \phi .
$$

If the company pays out $\varepsilon$ as dividends, then the initial capital reduces from $x$ to $x-\varepsilon$. Using the optimal strategy afterwards, so $V(x) \geq V(x-\varepsilon)+\varepsilon$. Subtracting $V(x-\varepsilon)$ from both sides, dividing by $\varepsilon$, and letting $\varepsilon \rightarrow 0$, we get

$$
V^{\prime}(x) \geq 1 \text {. }
$$

One of the inequalities (69), (70), and (71) is always tight (refer to Fleming and Soner [11]). $[0, \infty)$

Thus we derive the HJB equation satisfied by $V(x)$ on

$$
\begin{aligned}
\max \left\{\operatorname { s u p } _ { z \in R _ { + } } \left\{c V^{\prime}(x)+\lambda \int_{0}^{x+z} V(x-y) \mathrm{d} F(y)\right.\right. \\
\left.-(\lambda+\delta) V(x)\}, 1-V^{\prime}(x), V^{\prime}(x)-\phi\right\}=0 .
\end{aligned}
$$


To maximize $\int_{0}^{x+z} V(x-y) \mathrm{d} F(y)$, let us recall the proof of $z^{*}=V(0) / \phi$ in Section 2.2. We can find that $z^{*}$ is independent of $u_{0}$. So we also have the optimal lower capital injection barrier

$$
-z^{*}=-\frac{V(0)}{\phi}
$$

Hence when $x<0, V(x)$ can be expressed by

$$
V(x)= \begin{cases}0 & \text { if } x \leq-z^{*} \\ V(0)+\phi x & \text { if }-z^{*}<x<0 .\end{cases}
$$

In Section 2.2, the optimal dividend strategy and the optimal capital injection strategy are both barrier strategies under the assumption that $V(x)$ is concave on $(0, \infty)$. Moreover, the optimal dividend barrier $b$ and the upper optimal capital injection barrier $a$ are both independent of $u_{0}$. Here if $V(x)$ is concave on $(0, \infty)$, similar to discussion in Section 2.2, we can define the optimal dividend barrier $b:=\inf \left\{x: V^{\prime}(x) \leq 1\right\}$ and the optimal upper capital injection barrier $a:=\sup \{x$ : $\left.V^{\prime}(x) \geq \phi\right\} \vee 0$. And also $V(x)$ is continuously differentiable.

Proposition 9. If $V(x)$ is concave on $(0, \infty)$, the optimal upper capital injection barrier $a=0$.

Proof. The proof is similar as in Proposition 3, so we omit it here.

Now define a strategy $\pi^{1}=\left(D^{1}, Z^{1}\right)$ as follows:

$$
\begin{aligned}
& D_{0}^{1}=\max (x-b, 0) \\
& D_{t}^{1}=D_{0}^{1}+\int_{0}^{t} c 1_{\left\{X_{s}^{\pi^{1}}=b\right\}} \mathrm{d} s, \text { for } t>0, \\
& Z_{t}^{1}=\max \left\{-\inf _{0 \leq s<t}\left(X_{s}-D_{s}^{1}\right), 0\right\} \quad \text { for } t>0 .
\end{aligned}
$$

Let $T^{*}=\inf \left\{t \geq 0: X_{t}^{\pi^{1}} \leq-z^{*}\right\}$. Define strategy $\pi^{*}=$ $\left(D^{*}, Z^{*}\right)$ by the strategy $\pi^{1}$ stopped at $T^{*}$ :

$$
D_{t}^{*}=\left\{\begin{array}{ll}
D_{t}^{1}, & \text { if } t<T^{*}, \\
D_{T^{*}-}^{1}, & \text { if } t \geq T^{*},
\end{array} \quad Z_{t}^{*}= \begin{cases}Z_{t}^{1}, & \text { if } t<T^{*}, \\
Z_{T^{*}}^{1}, & \text { if } t \geq T^{*}\end{cases}\right.
$$

Under $\pi^{*}$, if the initial capital $x>b, x-b$ will be paid to the shareholders as dividends immediately. When the reserve process takes value in $(0, b)$, insurance company dose not pay dividend and shareholders do not inject capital. When the process reaches the barrier $b$, the premium income will be paid as dividends. If deficit occurs and it is less than $z^{*}$, the shareholders inject capital to recover the reserve process to 0 . Otherwise, they refuse to inject any capital and ruin occurs. $X_{t}^{*}=X_{t}-D_{t}^{*}+Z_{t}^{*}$ is the corresponding reserve process.

Theorem 10. If $V(x)$ is concave on $(0, \infty)$, the strategy $\pi^{*}$ defined in (76) is optimal; that is,

$$
V^{\pi^{*}}(x)=V(x)
$$

Proof. Note that $V^{\prime}\left(X_{t}^{*}\right)=V^{\prime}(b)=1$ on $\left\{X_{t}^{*}=b\right\}$. According to (76), the possible increment of $\left\{Z_{t}^{*}\right\}$ is at the time of claim arrivals. As in Lemma 5,

$$
\begin{aligned}
& V\left(X_{t \wedge T^{*}}^{*}\right) e^{-\delta\left(t \wedge T^{*}\right)} \\
& =V(x)-D_{0}^{1}+\phi \int_{0}^{t \wedge T^{*}} e^{-\delta s} \mathrm{~d} Z_{s}^{*} \\
& +\sum_{i=1}^{N_{t \wedge T^{*}}}\left[V\left(X_{T_{i^{-}}}^{*}-Y_{i}\right)-V\left(X_{T_{i}-}^{*}\right)\right] e^{-\delta T_{i}} \\
& +\sum_{i=1}^{N_{t \wedge T^{*}}}\left[V\left(X_{T_{i}-}^{*}\right) e^{-\delta T_{i}}-V\left(X_{T_{i-1}+}^{*}\right) e^{-\delta T_{i-1}}\right] \\
& +V\left(X_{t \wedge T^{*}}^{*}\right) e^{-\delta\left(t \wedge T^{*}\right)}-V\left(X_{T_{N_{t \wedge T^{*}}}^{*}}\right) e^{-\delta T_{N_{t \wedge T^{*}}}} \\
& =V(x)-D_{0}^{1}+\phi \int_{0}^{t \wedge T^{*}} e^{-\delta s} \mathrm{~d} Z_{s}^{*} \\
& +\sum_{i=1}^{N_{t \wedge T^{*}}}\left[V\left(X_{T_{i^{-}}}^{*}-Y_{i}\right)-V\left(X_{T_{i}-}^{*}\right)\right] e^{-\delta T_{i}} \\
& +\sum_{i=1}^{N_{t \wedge T^{*}}} \int_{T_{i-1}+}^{T_{i}-}\left[c V^{\prime}\left(X_{s}^{*}\right)-\delta V\left(X_{s}^{*}\right)\right] 1_{\left\{0<X_{s}^{*}<b\right\}} e^{-\delta s} \mathrm{~d} s \\
& -\sum_{i=1}^{N_{t \wedge T^{*}}} \int_{T_{i-1}+}^{T_{i}-} \delta V\left(X_{s}^{*}\right) 1_{\left\{X_{s}^{*}=b\right\}} e^{-\delta s} \mathrm{~d} s \\
& +\int_{T_{N_{t \wedge T^{*}}}}^{t \wedge T^{*}}\left[c V^{\prime}\left(X_{s}^{*}\right)-\delta V\left(X_{s}^{*}\right)\right] 1_{\left\{0<X_{s}^{*}<b\right\}} e^{-\delta s} \mathrm{~d} s
\end{aligned}
$$

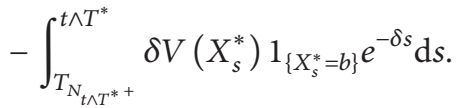

The process

$$
\begin{aligned}
& \left\{\sum_{i=1}^{N_{t \wedge T^{*}}}\left[V\left(X_{T_{i}-}^{*}-Y_{i}\right)-V\left(X_{T_{i}}^{*}\right)\right] e^{-\delta T_{i}}-\lambda\right. \\
& \left.\times \int_{0}^{t \wedge T^{*}} e^{-\delta s}\left[\int_{0}^{X_{s}^{*}+z^{*}} V\left(X_{s^{-}}^{*}-y\right) \mathrm{d} F(y)-V\left(X_{s-}^{*}\right)\right] \mathrm{d} s\right\}
\end{aligned}
$$

is a martingale with expected value 0 . Equivalently,

$$
\begin{gathered}
\left\{V\left(X_{t \wedge T^{*}}^{*}\right) e^{-\delta\left(t \wedge T^{*}\right)}-V(x)+D_{0}^{1}-\phi \int_{0}^{t \wedge T^{*}} e^{-\delta s} \mathrm{~d} Z_{s}^{*}\right. \\
-\int_{0}^{t \wedge T^{*}}\left[c V^{\prime}\left(X_{s}^{*}\right)+\lambda \int_{0}^{X_{s}^{*}+z^{*}} V\left(X_{s}^{*}-y\right) \mathrm{d} F(y)\right. \\
\left.-(\lambda+\delta) V\left(X_{s}^{*}\right)\right] 1_{\left\{0<X_{s}^{*}<b\right\}} e^{-\delta s} \mathrm{~d} s
\end{gathered}
$$




$$
\begin{aligned}
&-\int_{0}^{t \wedge T^{*}}\left[\lambda \int_{0}^{X_{s}^{*}+z^{*}} V\left(X_{s}^{*}-y\right) \mathrm{d} F(y)\right. \\
&\left.\left.-(\lambda+\delta) V\left(X_{s}^{*}\right)\right] 1_{\left\{X_{s}^{*}=b\right\}} e^{-\delta s} \mathrm{~d} s\right\}
\end{aligned}
$$

is a martingale. Because $V(x)$ is concave on $(0, \infty)$, the derivatives of $V(x)$ from left and right exist. Moreover, $F(y)$ is continuous, so $V(x)$ in (72) is continuously differentiable. For $V^{\prime}\left(X_{s}^{*}\right)>1$ on $\left\{0<X_{s}^{*}<b\right\}$, the first term on the lefthand side of (72) is 0 , thus the integral over $\left\{0<X_{s}^{*}<b\right\}$ on the expression above is 0 . Furthermore, from $V^{\prime}\left(X_{s}^{*}\right)=1$ on $\left\{X_{s}^{*}=b\right\}$ and (72), it follows that

$$
\lambda \int_{0}^{X_{s}^{*}+z^{*}} V\left(X_{s}^{*}-y\right) \mathrm{d} F(y)-(\lambda+\delta) V\left(X_{s}^{*}\right)=-c .
$$

Taking this expression into (80), we have

$$
\begin{aligned}
& \left\{V\left(X_{t \wedge T^{*}}^{*}\right) e^{-\delta\left(t \wedge T^{*}\right)}-V(x)+D_{0}^{1}\right. \\
& \left.-\phi \int_{0}^{t \wedge T^{*}} e^{-\delta s} \mathrm{~d} Z_{s}^{*}+\int_{0}^{t \wedge T^{*}} c 1_{\left\{X_{s}^{*}=b\right\}} e^{-\delta s} \mathrm{~d} s\right\}
\end{aligned}
$$

is a martingale with expected value 0 . Then

$$
\begin{gathered}
V(x)=E_{x}\left[V\left(X_{t \wedge T^{*}}^{*}\right) e^{-\delta\left(t \wedge T^{*}\right)}-\phi \int_{0}^{t \wedge T^{*}} e^{-\delta s} \mathrm{~d} Z_{s}^{*}\right. \\
\left.+\int_{0}^{t \wedge T^{*}} c 1_{\left\{X_{s}^{*}=b\right\}} e^{-\delta s} \mathrm{~d} s+D_{0}^{1}\right] .
\end{gathered}
$$

Note that

$$
\begin{aligned}
E_{x}\left[V\left(X_{t \wedge T^{*}}^{*}\right) e^{-\delta\left(t \wedge T^{*}\right)}\right] & =e^{-\delta t} E_{x}\left[V\left(X_{t}^{*}\right) 1_{\left(t \leq T^{*}\right)}\right] \\
& \leq e^{-\delta t} V(b)
\end{aligned}
$$

By the bounded convergence theorem,

$$
\lim _{t \rightarrow \infty} E_{x}\left[V\left(X_{t \wedge T^{*}}^{*}\right) e^{-\delta\left(t \wedge T^{*}\right)}\right]=0 .
$$

So

$V(x)$

$$
\begin{aligned}
& =\lim _{t \rightarrow \infty} E_{x}\left[\int_{0}^{t \wedge T^{*}} c 1_{\left\{X_{s}^{*}=b\right\}} e^{-\delta s} \mathrm{~d} s-\phi \int_{0}^{t \wedge T^{*}} e^{-\delta s} \mathrm{~d} Z_{s}^{*}+D_{0}^{1}\right] \\
& =E_{x}\left[\int_{0-}^{T^{*}-} e^{-\delta s} \mathrm{~d} D_{s}^{*}-\phi \int_{0}^{T^{*}} e^{-\delta s} \mathrm{~d} Z_{s}^{*}\right]=V^{*}(x) .
\end{aligned}
$$

3.2. Characterization of the Solution. How to characterize the solution $V(x)$ among other possible solutions?

Theorem 11. $V(x)$ is the minimal nonnegative solution to (72).

Proof. Let $f$ be a nonnegative solution to the HJB equation (72). $f$ is increasing because $f^{\prime}(x) \geq 1 .\left\{X_{t}^{*}\right\}$ is the reserve process under $\pi^{*}$. From Theorem 10

$$
\begin{gathered}
\left\{f\left(X_{t \wedge T^{*}}^{*}\right) e^{-\delta\left(t \wedge T^{*}\right)}-f(x)+D_{0}^{1}-\phi \int_{0}^{t \wedge T^{*}} e^{-\delta s} \mathrm{~d} Z_{s}^{*}\right. \\
-\int_{0}^{t \wedge T^{*}}\left[c f^{\prime}\left(X_{s}^{*}\right)+\lambda \int_{0}^{X_{s}^{*}+f(0) / \phi} f\left(X_{s}^{*}-y\right) \mathrm{d} F(y)\right. \\
\left.-(\lambda+\delta) f\left(X_{s}^{*}\right)\right] 1_{\left\{0<X_{s}^{*}<b\right\}} e^{-\delta s} \mathrm{~d} s \\
-\int_{0}^{t \wedge T^{*}}\left[\lambda \int_{0}^{X_{s}^{*}+f(0) / \phi} f\left(X_{s}^{*}-y\right) \mathrm{d} F(y)\right. \\
\left.\left.-(\lambda+\delta) f\left(X_{s}^{*}\right)\right] 1_{\left\{X_{s}^{*}=b\right\}} e^{-\delta s} \mathrm{~d} s\right\}
\end{gathered}
$$

is a martingale with expected value $0 . f(x)$ satisfies (72); then

$$
\begin{gathered}
c f^{\prime}\left(X_{s}^{*}\right)+\lambda \int_{0}^{X_{s}^{*}+f(0) / \phi} f\left(X_{s}^{*}-y\right) \mathrm{d} F(y) \\
-(\lambda+\delta) f\left(X_{s}^{*}\right) \leq 0 .
\end{gathered}
$$

Because $f^{\prime}(x) \geq 1$,

$$
\begin{aligned}
& \lambda \int_{0}^{X_{s}^{*}+f(0) / \phi} f\left(X_{s}^{*}-y\right) \mathrm{d} F(y)-(\lambda+\delta) f\left(X_{s}^{*}\right) \\
& \quad \leq-c f^{\prime}\left(X_{s}^{*}\right) \leq-c .
\end{aligned}
$$

From the non-negative property of $f(x)$, we have

$$
\begin{aligned}
f(x) \geq E_{x}\left[f\left(X_{t \wedge T^{*}}^{*}\right) e^{-\delta\left(t \wedge T^{*}\right)}-\phi \int_{0}^{t \wedge T^{*}} e^{-\delta s} \mathrm{~d} Z_{s}^{*}\right. \\
\left.+\int_{0}^{t \wedge T^{*}} c 1_{\left\{X_{s}^{*}=b\right\}} e^{-\delta s} \mathrm{~d} s+D_{0}^{1}\right] \\
\geq E_{x}\left[\int_{0-}^{t \wedge T^{*}-} e^{-\delta s} \mathrm{~d} D_{s}^{*}-\phi \int_{0}^{t \wedge T^{*}} e^{-\delta s} \mathrm{~d} Z_{s}^{*}\right] \\
=V^{*}(x)=V(x) .
\end{aligned}
$$

\section{Optimal Dividend and Capital Injection Strategies for Exponential Claims}

In this section we will consider the case that the claim size is exponentially distributed and the dividend strategy without restriction. Let $F(x)=1-e^{-\alpha x}$. 
First, we assume that $f(x)$ is an increasing, continuously differentiable and concave solution to the HJB equation (72) on $[0, \infty)$. Define $b=\inf \left\{x: f^{\prime}(x)=1\right\} \vee 0$. On $[0, b], f(x)$ satisfies

$$
\begin{aligned}
& c f^{\prime}(x)+\lambda \int_{0}^{x} f(x-y) \alpha e^{-\alpha y} \mathrm{~d} y \\
& \quad+\lambda \int_{x}^{x+f(0) / \phi}[f(0)+\phi(x-y)] \alpha e^{-\alpha y} \mathrm{~d} y \\
& \quad-(\lambda+\delta) f(x)=0 .
\end{aligned}
$$

Let $z=x-y$ and change (91) into

$$
\begin{aligned}
& c f^{\prime}(x)+\lambda e^{-\alpha x} \int_{0}^{x} f(z) \alpha e^{\alpha z} \mathrm{~d} z \\
& +\lambda e^{-\alpha x} \int_{-f(0) / \phi}^{0}[f(0)+\phi z] \alpha e^{\alpha z} \mathrm{~d} z \\
& -(\lambda+\delta) f(x)=0 .
\end{aligned}
$$

The above expression can be derivative, so it yields

$$
\begin{gathered}
c f^{\prime \prime}(x)-\alpha \lambda e^{-\alpha x} \int_{0}^{x} f(z) \alpha e^{\alpha z} \mathrm{~d} z+\alpha \lambda f(x) \\
-\alpha \lambda e^{-\alpha x} \int_{-f(0) / \phi}^{0}[f(0)+\phi z] \alpha e^{\alpha z} \mathrm{~d} z \\
-(\lambda+\delta) f^{\prime}(x)=0 .
\end{gathered}
$$

Combining (92) with (93), we get the differentiable equation about $f(x)$

$$
c f^{\prime \prime}(x)+(\alpha c-(\lambda+\delta)) f^{\prime}(x)-\alpha \delta f(x)=0 .
$$

Its solution is

$$
f(x)=C_{1} e^{v_{1} x}+C_{2} e^{v_{2} x},
$$

where $v_{1}, v_{2}$ are the solutions of equation $c v^{2}+(\alpha c-(\lambda+\delta)) v-$ $\alpha \delta=0$; that is,

$$
\begin{aligned}
& v_{1}=\frac{\lambda+\delta-\alpha c-\sqrt{(\lambda+\delta-\alpha c)^{2}+4 \alpha c \delta}}{2 c}<0, \\
& v_{2}=\frac{\lambda+\delta-\alpha c+\sqrt{(\lambda+\delta-\alpha c)^{2}+4 \alpha c \delta}}{2 c}>0 .
\end{aligned}
$$

When $x \geq b$, we conjecture that

$$
f(x)=x-b+f(b), \quad x \geq b .
$$

Therefore, from (95) and (97), the suggested solution of HJB equation (72) has the form

$$
f(x)= \begin{cases}C_{1} e^{v_{1} x}+C_{2} e^{v_{2} x} & \text { if } 0 \leq x \leq b, \\ x-b+C_{1} e^{v_{1} b}+C_{2} e^{v_{2} b} & \text { if } x \geq b,\end{cases}
$$

where $C_{1}, C_{2}$, and $b$ are to be determined later.
Lemma 12. At $x=b$, we have

$$
f^{\prime \prime}(b)=0, \quad f(b)=\frac{\alpha c-\lambda-\delta}{\alpha \delta} .
$$

Proof. As we have assumed that $f(x)$ satisfies HJB equation (72), when $x>b$, we have

$$
c+\lambda \int_{0}^{x+f(0) / \phi} f(x-y) \alpha e^{-\alpha y} \mathrm{~d} y-(\lambda+\delta) f(x) \leq 0 .
$$

Note that

$$
\begin{aligned}
\int_{0}^{x+} & f(0) / \phi \\
= & \int_{0}^{x-b}\left[f(b)+(x-y) \alpha e^{-\alpha y} \mathrm{~d} y\right. \\
& +\int_{x-b}^{x+f(0) / \phi} f(x-y) \alpha e^{-\alpha y} \mathrm{~d} y \\
= & {[f(b)+(x-b)]\left(1-e^{-\alpha(x-b)}\right)-\int_{0}^{x-b} y \alpha e^{-\alpha y} \mathrm{~d} y } \\
& +\int_{0}^{b+f(0) / \phi} f(b-u) \alpha e^{-\alpha(x-b+u)} \mathrm{d} u \\
= & {[f(b)+(x-b)]\left(1-e^{-\alpha(x-b)}\right)+(x-b) e^{-\alpha(x-b)} } \\
& -\frac{1}{\alpha}+\frac{1}{\alpha} e^{-\alpha(x-b)} \\
& +\left(\int_{0}^{b+f(0) / \phi} f(b-u) \alpha e^{-\alpha u} \mathrm{~d} u\right) e^{-\alpha(x-b)}
\end{aligned}
$$

and from the HJB equation (72), when $x=b$,

$$
c+\lambda \int_{0}^{b+f(0) / \phi} f(b-y) \alpha e^{-\alpha y} \mathrm{~d} y-(\lambda+\delta) V(b)=0 .
$$

Plugging (101) and (102) into the left side of (100), then we can rewrite the expression by

$$
\begin{aligned}
c+\lambda\{ & {[f(b)+(x-b)]\left(1-e^{-\alpha(x-b)}\right) } \\
& \left.+(x-b) e^{-\alpha(x-b)}-\frac{1}{\alpha}+\frac{1}{\alpha} e^{-\alpha(x-b)}\right\} \\
& +[(\lambda+\delta) f(b)-c] e^{-\alpha(x-b)}-(\lambda+\delta)(f(b)+(x-b)) \\
= & \left(c-\delta f(b)-\frac{\lambda}{\alpha}\right)\left(1-e^{-\alpha(x-b)}\right)-\delta(x-b) .
\end{aligned}
$$

Therefore (100) is established if and only if $f(b) \geq[\alpha c-\lambda-$ $\left.\alpha \delta(x-b) /\left(1-e^{-\alpha(x-b)}\right)\right] /(\alpha \delta)$ for all $x>b$. When $x \rightarrow b$, $f(b) \geq(\alpha c-\lambda-\delta) /(\alpha \delta)$. 

that

In (94), let $x=b$. From $f(b) \geq(\alpha c-\lambda-\delta) /(\alpha \delta)$, we find

$$
\begin{aligned}
0 & =c f^{\prime \prime}(b)+(\alpha c-(\lambda+\delta))-\alpha \delta f(b) \\
& \leq c f^{\prime \prime}(b)+(\alpha c-(\lambda+\delta))-\alpha \delta \frac{\alpha c-\lambda-\delta}{\alpha \delta}=c f^{\prime \prime}(b),
\end{aligned}
$$

which implies $f^{\prime \prime}(b) \geq 0$.

On the other hand, because $f(x)$ is concave, we have $f^{\prime \prime}(x) \leq 0$. Particularly, $f^{\prime \prime}(b) \leq 0$. Combining the discussion above, $f^{\prime \prime}(b)=0$.

Furthermore, taking $f^{\prime \prime}(b)=0$ and $f^{\prime}(b)=1$ into (94) yields

$$
f(b)=\frac{\alpha c-\lambda-\delta}{\alpha \delta} .
$$

Next we will determine $C_{1}, C_{2}$, and $b$.

From the expression of $f(x)$ in $(95)$ and $f^{\prime \prime}(b)=0$ (it has been proved in Lemma 12), it holds that

$$
f^{\prime \prime}(b)=C_{1} v_{1}^{2} e^{v_{1} b}+C_{2} v_{2}^{2} e^{v_{2} b}=0 .
$$

The continuously differentiable property of $f(x)$ tells us that

$$
f^{\prime}(b)=C_{1} v_{1} e^{v_{1} b}+C_{2} v_{2} e^{v_{2} b}=1 .
$$

Combining the two equations above, we can get the expression of $C_{1}$ and $C_{2}$ :

$$
C_{1}=\frac{v_{2}}{\left(v_{2}-v_{1}\right) v_{1} e^{v_{1} b}}, \quad C_{2}=\frac{v_{1}}{\left(v_{1}-v_{2}\right) v_{2} e^{v_{2} b}} .
$$

When $x=0$, (95) informs us that $f(0)=C_{1}+C_{2}, f^{\prime}(0)=$ $C_{1} v_{1}+C_{2} v_{2}$. Meanwhile, at $x=0$ the integral-differential equation (91) implies

$$
c f^{\prime}(0)+\lambda \int_{0}^{f(0) / \phi}[f(0)-\phi y] \mathrm{d} F(y)-(\lambda+\delta) f(0)=0 .
$$

Together with (108), (109) can be rewritten as

$$
\begin{aligned}
\frac{c v_{2}}{v_{2}-v_{1}} e^{-v_{1} b}+\frac{c v_{1}}{v_{1}-v_{2}} e^{-v_{2} b} & \\
- & \frac{\lambda \phi}{\alpha}\left(1-e^{-(\alpha / \phi)\left[\left(v_{2} /\left(v_{2}-v_{1}\right) v_{1}\right) e^{-v_{1} b}+\left(v_{1} /\left(v_{1}-v_{2}\right) v_{2}\right) e^{-v_{2} b}\right]}\right) \\
- & \delta\left[\frac{v_{2}}{\left(v_{2}-v_{1}\right) v_{1}} e^{-v_{1} b}+\frac{v_{1}}{\left(v_{1}-v_{2}\right) v_{2}} e^{-v_{2} b}\right]=0,
\end{aligned}
$$

which can be used to calculate $b$.

Proposition 13. The solution of (110) is unique. $b=0$ if and only if

$$
\lambda+\delta \geq \lambda \phi\left(1-e^{-(\alpha c-(\lambda+\delta)) / \phi \delta}\right) .
$$

Proof. To analyse the solution of (110), we first define a function

$$
\begin{aligned}
g(z):= & \frac{c v_{2}}{v_{2}-v_{1}} e^{-v_{1} z}+\frac{c v_{1}}{v_{1}-v_{2}} e^{-v_{2} z} \\
& -\frac{\lambda \phi}{\alpha}\left(1-e^{-(\alpha / \phi)\left[\left(v_{2} /\left(v_{2}-v_{1}\right) v_{1}\right) e^{-v_{1} z}+\left(v_{1} /\left(v_{1}-v_{2}\right) v_{2}\right) e^{-v_{2} z}\right]}\right) \\
& -\delta\left[\frac{v_{2}}{\left(v_{2}-v_{1}\right) v_{1}} e^{-v_{1} z}+\frac{v_{1}}{\left(v_{1}-v_{2}\right) v_{2}} e^{-v_{2} z}\right],
\end{aligned}
$$

where $z \geq 0$. In view of $v_{1}+v_{2}=[(\lambda+\delta)-\alpha c] / c$ and $v_{1} v_{2}=$ $-\alpha \delta / c$, we find that

$$
\begin{aligned}
g^{\prime}(z)= & c \frac{v_{1} v_{2}}{v_{1}-v_{2}}\left(e^{-v_{1} z}-e^{-v_{2} z}\right) \\
& +\delta\left(\frac{v_{2}}{v_{2}-v_{1}} e^{-v_{1} z}+\frac{v_{1}}{v_{1}-v_{2}} e^{-v_{2} z}\right) \\
& -\lambda\left(\frac{v_{2}}{v_{1}-v_{2}} e^{-v_{1} z}+\frac{v_{1}}{v_{2}-v_{1}} e^{-v_{2} z}\right) \\
& \cdot e^{-(\alpha / \phi)\left[\left(v_{2} /\left(v_{2}-v_{1}\right) v_{1}\right) e^{-v_{1} z}+\left(v_{1} /\left(v_{1}-v_{2}\right) v_{2}\right) e^{-v_{2} z}\right]}>0,
\end{aligned}
$$

which implies $g(z)$ is increasing strictly. So the solution is unique. Consider

$$
\begin{aligned}
\lim _{z \rightarrow \infty} g(z)=\lim _{z \rightarrow \infty}[ & \frac{c v_{2}}{v_{2}-v_{1}} e^{-v_{1} z} \\
& -\frac{\lambda \phi}{\alpha}\left(1-e^{-(\alpha / \phi)\left(v_{2} /\left(v_{2}-v_{1}\right) v_{1}\right) e^{-v_{1} z}}\right) \\
& \left.-\delta \frac{v_{2}}{\left(v_{2}-v_{1}\right) v_{1}} e^{-v_{1} z}\right]=\infty
\end{aligned}
$$

Hence $b=0$ if and only if $g(0) \geq 0$. While

$$
\begin{aligned}
g(0) & =c-\frac{\lambda \phi}{\alpha}\left(1-e^{-(\alpha / \phi)\left(\left(v_{1}+v_{2}\right) / v_{1} v_{2}\right)}\right)-\delta \frac{v_{1}+v_{2}}{v_{1} v_{2}} \\
& =\frac{\lambda+\delta}{\alpha}-\frac{\lambda \phi}{\alpha}\left(1-e^{-(\alpha c-(\lambda+\delta)) / \phi \delta}\right),
\end{aligned}
$$

so the necessary and sufficient condition of $b=0$ is $\lambda+\delta \geq$ $\lambda \phi\left(1-e^{-(\alpha c-(\lambda+\delta)) / \phi \delta}\right)$.

Based on the discussion above, we obtain the expression of $f(x)$. The following proposition will verify the concavity of $f(x)$.

Proposition 14. $f(x)$ is concave on $[0, \infty)$.

Proof. When $x \in[0, b)$, from (95) and (108), we have

$$
\begin{aligned}
f^{\prime \prime}(x) & =\frac{v_{2} v_{1}}{v_{2}-v_{1}} e^{v_{1}(x-b)}+\frac{v_{1} v_{2}}{v_{1}-v_{2}} e^{v_{2}(x-b)} \\
& =\frac{v_{2} v_{1}}{v_{2}-v_{1}}\left(e^{v_{1}(x-b)}-e^{v_{2}(x-b)}\right)<0
\end{aligned}
$$


due to the fact $v_{1}<0$ and $v_{2}>0$. What is more, $f^{\prime \prime}(x)=0$ for $x \geq b$. Therefore $f^{\prime \prime}(x) \leq 0$ on $[0, \infty)$. This establishes the concavity of $f(x)$ on $[0, \infty)$.

Proposition 15. $f(x)$ is the solution of HJB equation (72) when $x \in[0, \infty)$.

Proof. From the construction of $f(x)$,

$$
\begin{array}{r}
f^{\prime}(x)=1 \quad \text { for } x \geq b \\
c+\lambda \int_{0}^{x+f(0) / \phi} f(x-y) \alpha e^{-\alpha y} \mathrm{~d} y-(\lambda+\delta) f(x)=0 \\
\text { for } 0 \leq x \leq b
\end{array}
$$

are established obviously. We only remain to show that $f(x)$ satisfies

$$
\begin{gathered}
f^{\prime}(x)>1 \quad \text { for } 0 \leq x<b, \\
c+\lambda \int_{0}^{x+f(0) / \phi} f(x-y) \alpha e^{-\alpha y} \mathrm{~d} y-(\lambda+\delta) f(x)<0 \\
\quad \text { for } x>b, \\
f^{\prime}(x)<\phi \text { for } x>0 .
\end{gathered}
$$

From the concavity of $f(x)$ and $f^{\prime}(b)=1,(118)$ is true.

Similar to the proof in Lemma 12 we can show (119) is established.

To prove (120), according to the concavity of $f(x)$, we only need to show $f^{\prime}(0)<\phi$. Let $x=0$ in (91) and assume that $f^{\prime}(0) \geq \phi$. We find

$$
\begin{aligned}
f(0) & =\frac{\alpha c f^{\prime}(0)-\lambda \phi+\lambda \phi e^{-(\alpha / \phi) f(0)}}{\alpha \delta} \\
& \geq \frac{\alpha c \phi-\lambda \phi+\lambda \phi e^{-(\alpha / \phi) f(0)}}{\alpha \delta} \\
& =\phi \frac{\alpha c-\lambda+\lambda e^{-(\alpha / \phi) f(0)}}{\alpha \delta} \\
& >\phi \frac{\alpha c-\lambda-\delta}{\alpha \delta}=\phi f(b) .
\end{aligned}
$$

The last equality comes from $f(b)=(\alpha c-\lambda-\delta) / \alpha \delta$ which is proved in Lemma 12. While $f(0)>\phi f(b)$ is impossible because $f(x)$ is increasing and $\phi>1$. This also tells us that $f^{\prime}(0)<\phi$. Therefore $f^{\prime}(0) \leq \phi$. And the proof is completed.

The following theorem gives the optimal value function and optimal strategies when the claim size is exponentially distributed.

Theorem 16. Suppose $F(x)=1-e^{-\alpha x}$. The value function $V(x)$ and the optimal strategy are as follows.
(1) If $\lambda+\delta<\lambda \phi\left(1-e^{-(\alpha c-(\lambda+\delta)) / \phi \delta}\right)$, the value function

$$
V(x)= \begin{cases}0 & \text { if } x<-z^{*}, \\ V(0)+\phi x & \text { if }-z^{*} \leq x<0, \\ C_{1} e^{v_{1} x}+C_{2} e^{v_{2} x} & \text { if } 0 \leq x<b, \\ x-b+C_{1} e^{v_{1} b}+C_{2} e^{v_{2} b} & \text { if } x \geq b,\end{cases}
$$

where $C_{1}, C_{2}$ are given by (108). The optimal lower capital injection barrier $-z^{*}=-V(0) / \phi$ and the optimal upper capital injection barrier $a=0$. The optimal dividend barrier b can be calculated from (110).

(2) If $\lambda+\delta \geq \lambda \phi\left(1-e^{-(\alpha c-(\lambda+\delta)) / \phi \delta}\right)$, the value function

$V(x)$

$$
=\left\{\begin{aligned}
0 & \text { if } x<-z^{*}, \\
V(0)+\phi x & \\
=\left[\phi W\left(\frac{\lambda}{\delta} e^{-(\alpha c-\lambda \phi) / \phi \delta}\right)\right. & \\
\left.+\frac{\alpha c-\lambda \phi}{\delta}\right](\alpha+\phi x)^{-1} & \text { if }-z^{*} \leq x<0, \\
x+V(0) & \\
=x+\left[\phi W\left(\frac{\lambda}{\delta} e^{-(\alpha c-\lambda \phi) / \phi \delta}\right)\right. & \\
\left.+\frac{\alpha c-\lambda \phi}{\delta}\right](\alpha)^{-1} & \text { if } x \geq 0
\end{aligned}\right.
$$

where $W(x)$ is Lambert $W$ function which is the solution of $W(x) e^{W(x)}=x$. The optimal lower capital injection barrier $-z^{*}=-V(0) / \phi$ and the optimal upper capital injection barrier $a=0$. The optimal dividend barrier $b=0$.

Proof. (1) For $x \geq 0$, because $f(x)$ is the solution of HJB equation (72) on $[0, \infty)$, from Theorem 11, we know $V(x)$ coincides with $f(x)$ on $[0, \infty)$. Because $V(x)$ is concave on $[0, \infty)$, Proposition 9 and the expression (73) inform us what are the optimal upper and lower capital injection barriers. Under the condition in (1), $b>0$ by Proposition 13. $b$ can be derived by (110). When $x<0$, the expression of $V(x)$ has been discussed in (74). Therefore, (122) is established. Figure 1 shows us the sample path of the reserve process under the optimal strategy $\pi^{*}$ and Figure 2 is the figure of the value function $V(x)$.

(2) If $\lambda+\delta \geq \lambda \phi\left(1-e^{-(\alpha c-(\lambda+\delta)) / \phi \delta}\right)$, then $b=0$ by Proposition 13. $b=0$ means that under the optimal strategy, the shareholders will act as the insurer: they receive 


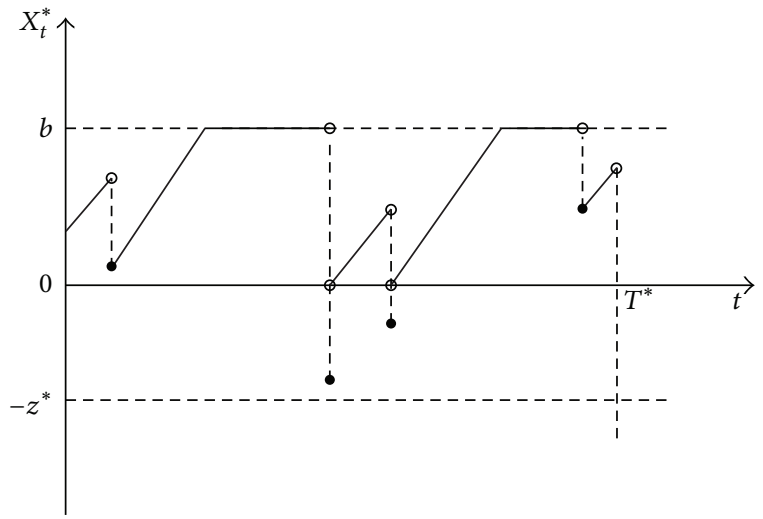

FIGURE 1: The sample path of the reserve process under the optimal strategy $\pi^{*}$.

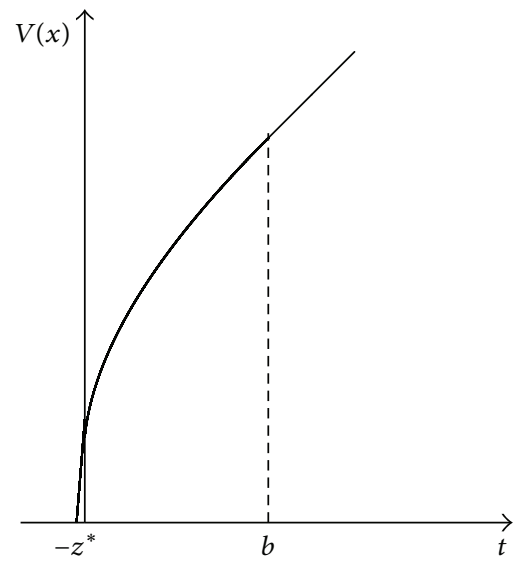

Figure 2: The value function $V(x)$.

the premium income and pay each claim in full when it occurs (see Dickson and Waters [7]). V(0) must be recalculated by

$$
\begin{aligned}
V(0)= & E\left[\int_{0}^{T_{1}} c e^{-\delta t} \mathrm{~d} t+e^{-\delta T_{1}}\left(V(0)-\phi Y_{1}\right) 1_{\left(Y_{1} \leq V(0) / \phi\right)}\right] \\
= & \int_{0}^{\infty} \lambda e^{-\lambda s} \int_{0}^{s} c e^{-\delta t} \mathrm{~d} t \mathrm{~d} s \\
& +\int_{0}^{\infty} \lambda e^{-\lambda s} e^{-\delta s} \int_{0}^{V(0) / \phi}(V(0)-\phi y) \alpha e^{\alpha y} \mathrm{~d} y \mathrm{~d} s \\
= & \frac{\alpha c-\lambda \phi}{\alpha \delta}+\frac{\lambda \phi}{\alpha \delta} e^{-\alpha(V(0) / \phi)} .
\end{aligned}
$$

So $V(0)=\left[\phi W\left((\lambda / \delta) e^{-(\alpha c-\lambda \phi) / \phi \delta}\right)+(\alpha c-\lambda \phi) / \delta\right] / \alpha$, where $W(x)$ is Lambert $W$ function which is the solution of $W(x) e^{W(x)}=x .-z^{*}=-V(0) / \phi$ and $a=0$ are same as the discussion in proof of (1). Therefore, $V(x)$ can be expressed by (123). Figure 3 is the figure of the value function $V(x)$.

Note that it is the first time that Lambert $W$ function is used in the risk theory. It simplifies the expression of $V(x)$ when $b=0$.

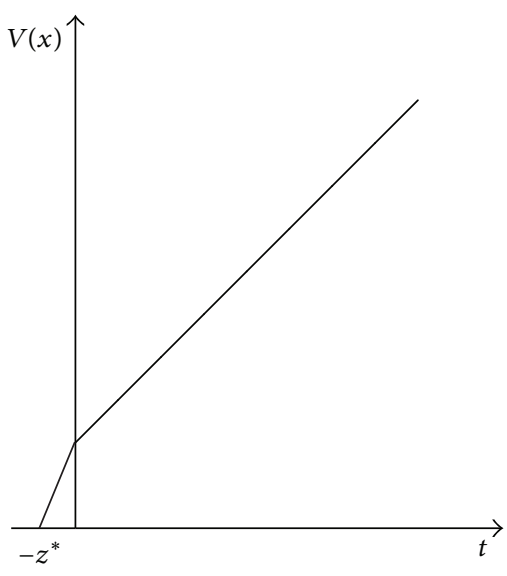

FIgURE 3: The value function $V(x)$.

\section{Conflict of Interests}

The authors declare that there is no conflict of interests regarding the publication of this paper.

\section{Acknowledgment}

This work has been supported by The Natural Science Foundation of China (Grant no. 11471218 and no. 71303047).

\section{References}

[1] B. Borch, "The rescue of an insurance company after ruin," ASTIN Bulletin, vol. 15, no. 2, pp. 280-292, 1968.

[2] S. P. Sethi and M. I. Taksar, "Optimal financing of a corporation subject to random returns," Mathematical Finance, vol. 12, no. 2, pp. 155-172, 2002.

[3] A. Løkka and M. Zervos, "Optimal dividend and issuance of equity policies in the presence of proportional costs," Insurance: Mathematics \& Economics, vol. 42, no. 3, pp. 954-961, 2008.

[4] L. He and Z. Liang, "Optimal financing and dividend control of the insurance company with proportional reinsurance policy," Insurance: Mathematics \& Economics, vol. 42, no. 3, pp. 976-983, 2008.

[5] L. He and Z. Liang, "Optimal financing and dividend control of the insurance company with fixed and proportional transaction costs," Insurance: Mathematics \& Economics, vol. 44, no. 1, pp. 88-94, 2009.

[6] N. Bäuerle, "Approximation of optimal reinsurance and dividend payout policies," Mathematical Finance, vol. 14, no. 1, pp. 99-113, 2004.

[7] D. C. Dickson and H. R. Waters, "Some optimal dividends problems," Astin Bulletin, vol. 34, no. 1, pp. 47-74, 2004.

[8] H. U. Gerber, E. S. Shiu, and N. Smith, "Maximizing dividends without bankruptcy," Astin Bulletin, vol. 36, no. 1, pp. 5-23, 2006.

[9] N. Kulenko and H. Schmidli, "Optimal dividend strategies in a Cramér-Lundberg model with capital injections," Insurance: Mathematics and Economics, vol. 43, no. 2, pp. 270-278, 2008.

[10] P. Azcue and N. Muler, "Optimal reinsurance and dividend distribution policies in the Cramér-Lundberg model," Mathematical Finance, vol. 15, no. 2, pp. 261-308, 2005. 
[11] W. H. Fleming and H. M. Soner, Controlled Markov Pro cesses and Viscosity Solutions, vol. 25 of Applications of Mathematics, Springer, New York, NY, USA, 1993.

[12] R. Radner and L. Shepp, "Risk vs. profit potential: a model for corporate strategy," Journal of Economic Dynamics and Control, vol. 20, no. 8, pp. 1373-1393, 1996.

[13] S. Asmussen and M. Taksar, "Controlled diffusion models for optimal dividend pay-out," Insurance: Mathematics \& Economics, vol. 20, no. 1, pp. 1-15, 1997.

[14] B. Højgaard and M. Taksar, "Optimal proportional reinsurance policies for diffusion models," Scandinavian Actuarial Journal, vol. 1998, no. 2, pp. 166-180, 1998.

[15] B. Højgaard and M. Taksar, "Controlling risk exposure and dividends payout schemes: insurance company example," Mathematical Finance, vol. 9, no. 2, pp. 153-182, 1999.

[16] C. Maglaras, "Discrete-review policies for scheduling stochastic networks: trajectory tracking and fluid-scale asymptotic optimality," The Annals of Applied Probability, vol. 10, no. 3, pp. 897929, 2000. 


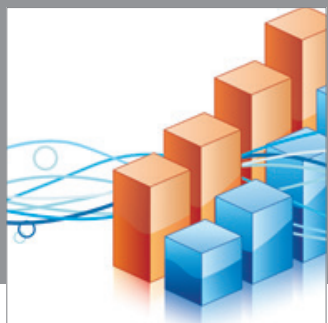

Advances in

Operations Research

mansans

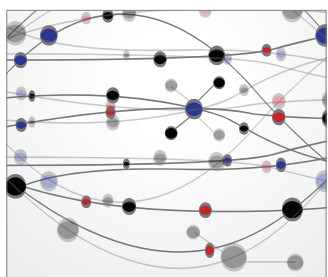

The Scientific World Journal
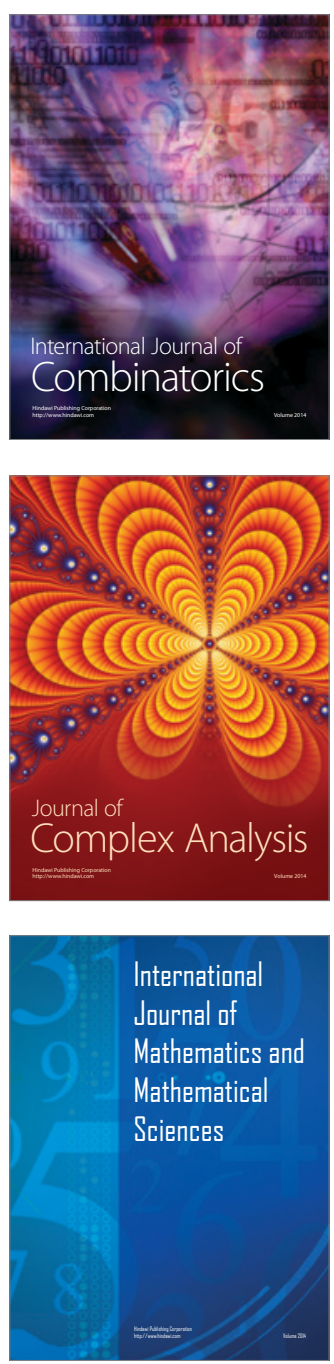
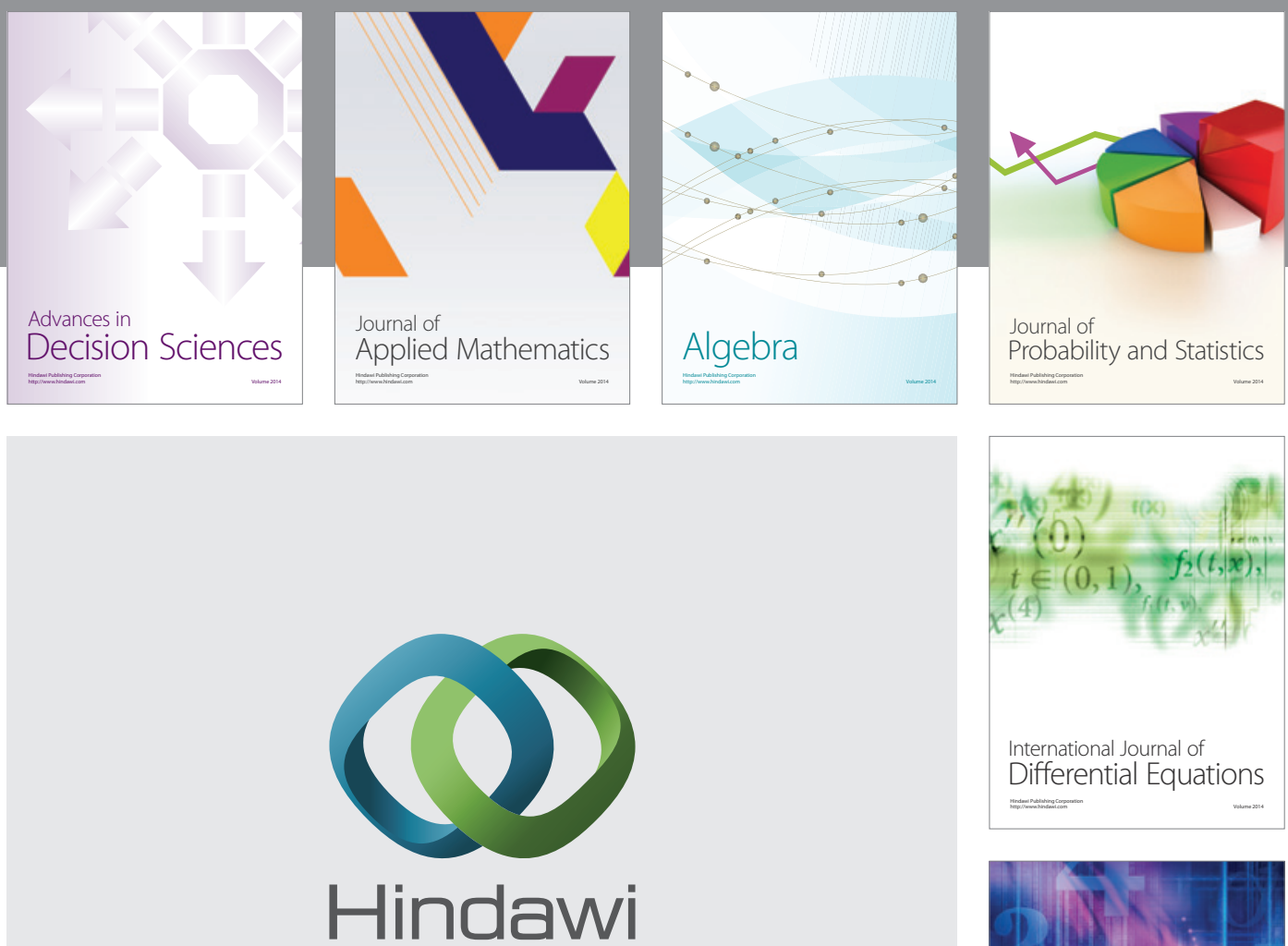

Submit your manuscripts at http://www.hindawi.com
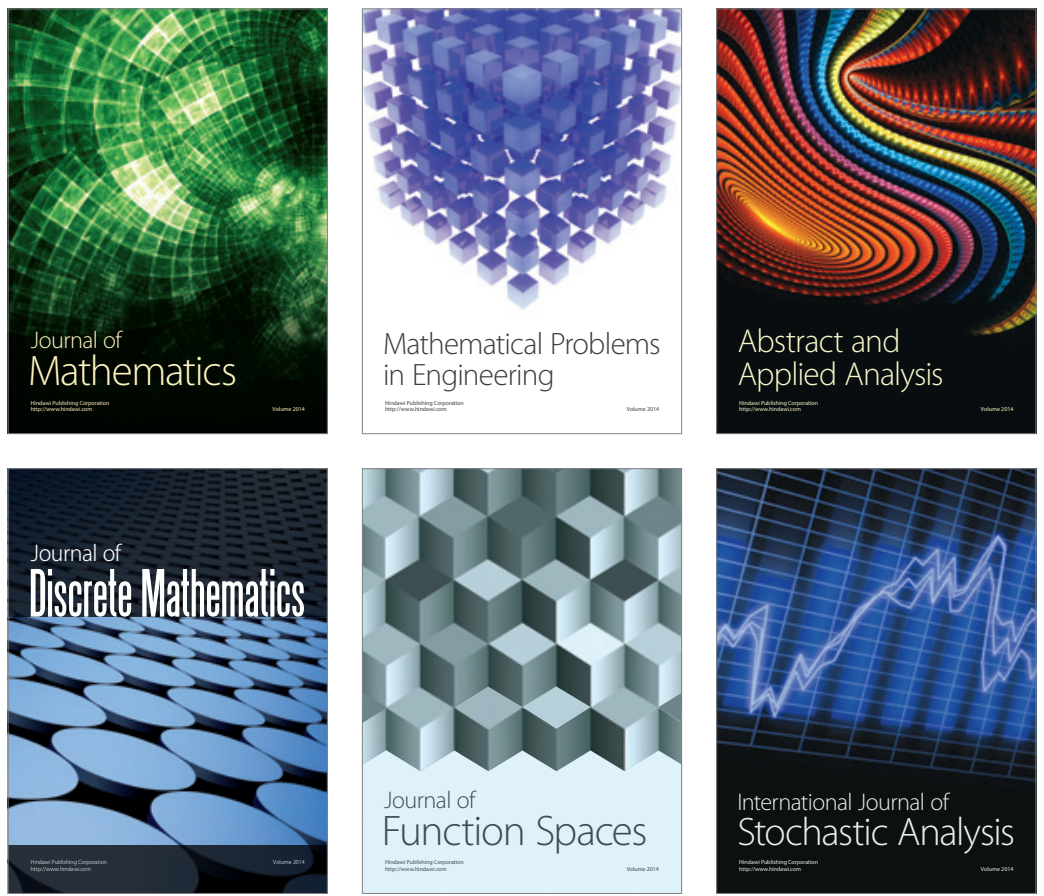

Journal of

Function Spaces

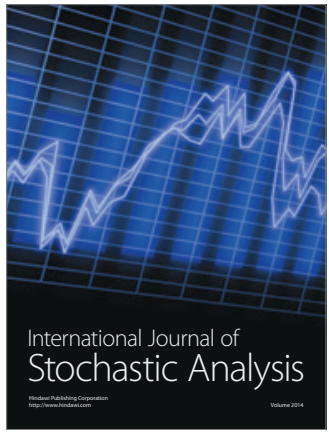

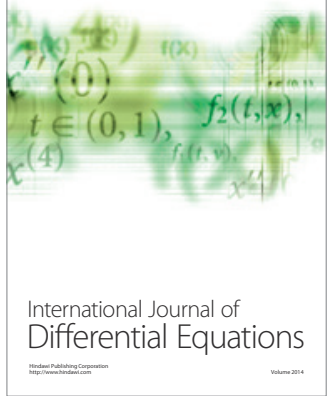
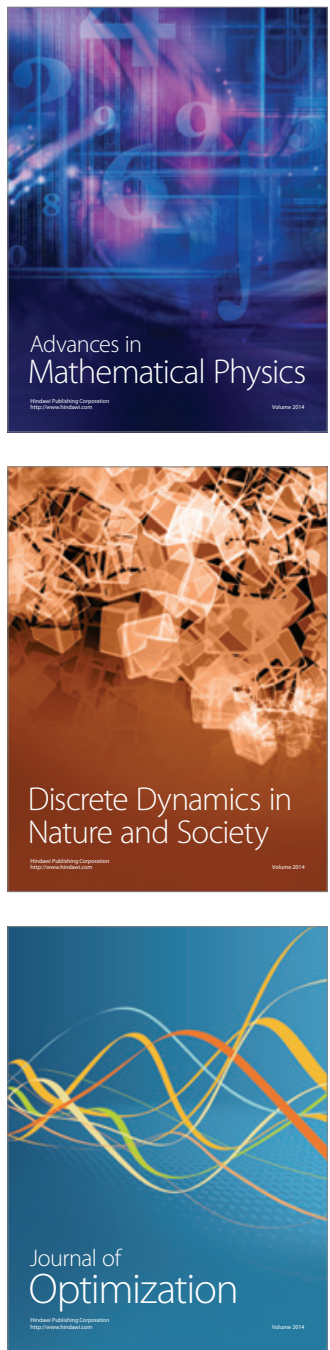